\title{
NMR and HPLC Spectra of the Compounds
}

\section{DNA Structural Alteration Leading to Antibacterial Properties of 6-Nitroquinoxaline Derivatives}

\author{
Khondakar Sayef Ahammed, ${ }^{\dagger, \S}$ Ritesh Pal, ${ }^{\dagger, \downarrow}{ }^{\star}$ Jeet Chakraborty, ${ }^{\dagger, \S}$ Ajay Kanungo, ${ }^{\dagger, \star}$ Polnati Sravani \\ Purnima, ${ }^{\dagger}$ Sanjay Dutta ${ }^{*} \dagger, *$
}

$†$ Organic and Medicinal Chemistry Division, CSIR- Indian Institute of Chemical Biology

4, Raja S.C. Mullick Road, Kolkata-700032, West Bengal, India.

E-mail: sanjaydutta@iicb.res.in

\$ Academy of Scientific and Innovative Research (AcSIR), Kolkata-700032, West Bengal, India.

§These authors contributed equally.

* Corresponding author. 


\section{Table of Contents}

Section S1: ${ }^{1} \mathrm{H}$ and ${ }^{13} \mathrm{C}$ Spectral data of compounds — Page S3-S22

Section S2: HPLC chromatogram of compounds - Page S23-S32. 
Section S1 : NMR Spectra $\left({ }^{1} \mathrm{H}\right.$ and $\left.{ }^{13} \mathrm{C}\right)$

${ }^{1} \mathrm{H}$ NMR of 3a (DMSO- $\left.\mathrm{d}_{6}, 600 \mathrm{MHz}\right)$ :-

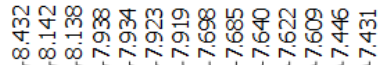

赵

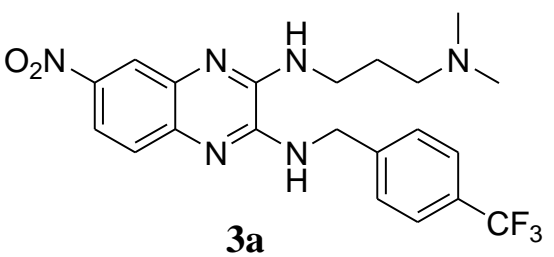

$3 \mathbf{a}$

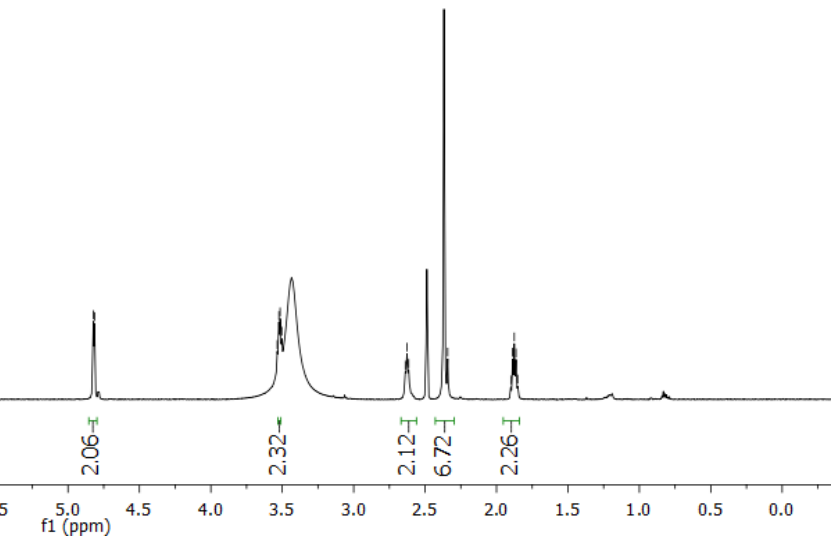

${ }^{13} \mathrm{C}$ NMR of 3a (DMSO-d $\left.6,150 \mathrm{MHz}\right)$ :-

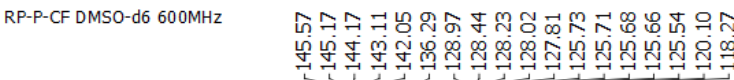
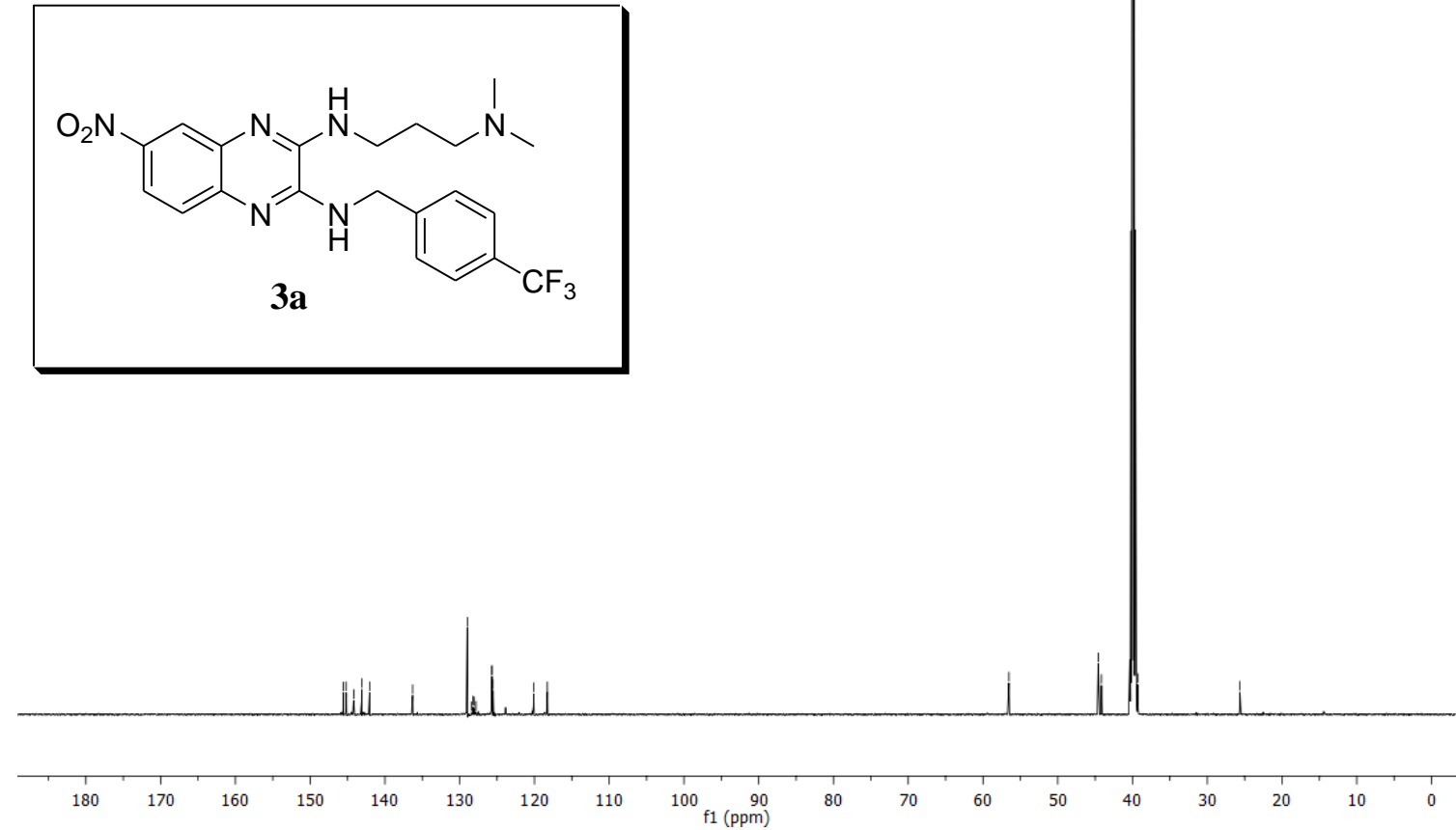
${ }^{1} \mathrm{H}$ NMR of $\mathbf{3 b}\left(\mathrm{CDCl}_{3}, 600 \mathrm{MHz}\right)$ :-

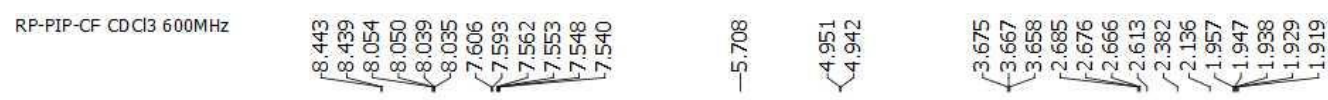<smiles>CN1CCN(CCCNc2nc3ccc([N+](=O)[O-])cc3nc2NCc2ccc(C(F)(F)F)cc2)CC1</smiles>

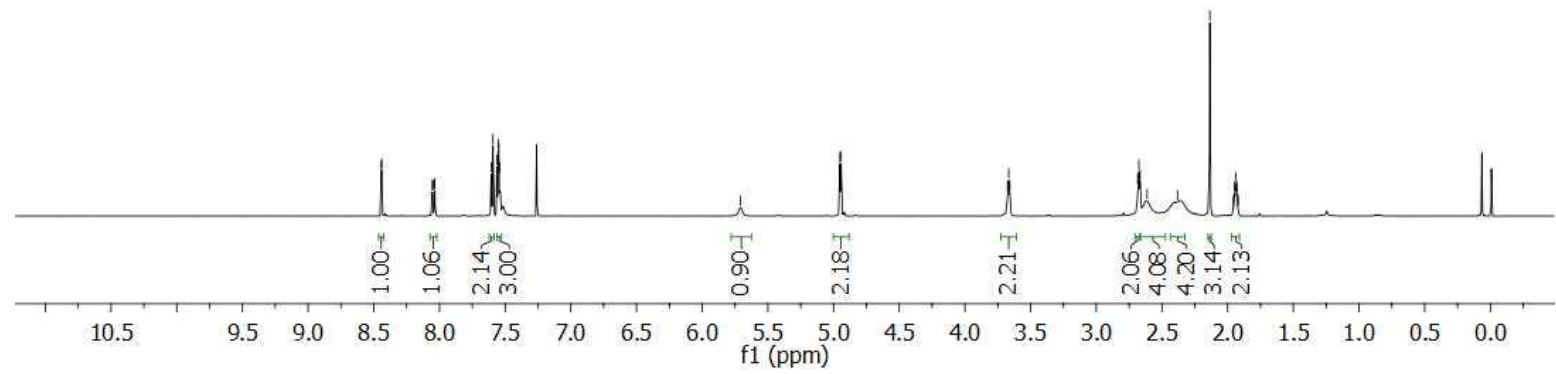

${ }^{13} \mathrm{C}$ NMR of $\mathbf{3 b}\left(\mathrm{CDCl}_{3}, 150 \mathrm{MHz}\right)$ :-

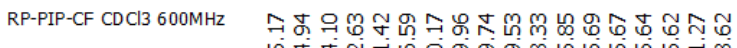

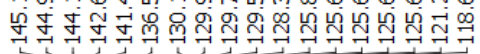

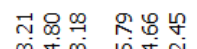

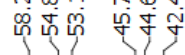

$\stackrel{\sim}{\stackrel{\sim}{\sim}}$<smiles>O=[N+]([O-])c1ccc2nc(NCc3ccc(C(F)(F)F)cc3)c(NCCCN3CCN(CCNCc4ccccc4)CC3)nc2c1</smiles>

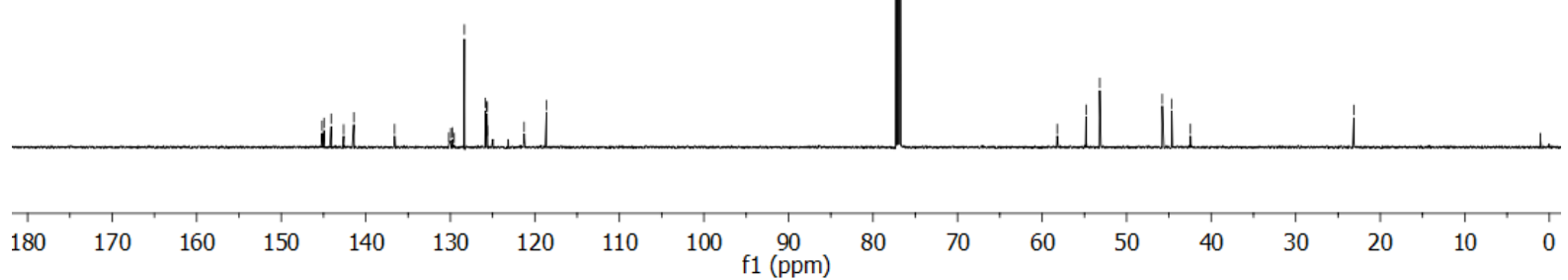


${ }^{1} \mathrm{H}$ NMR of $\mathbf{3 c}\left(\mathrm{CDCl}_{3}, 400 \mathrm{MHz}\right)$ :-

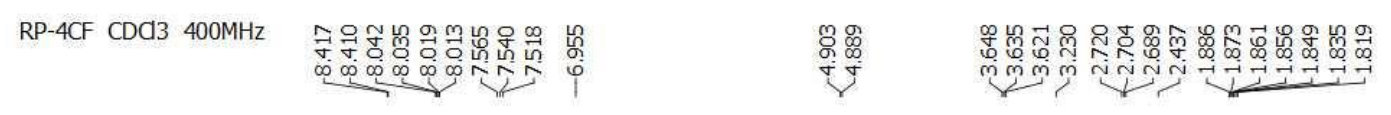
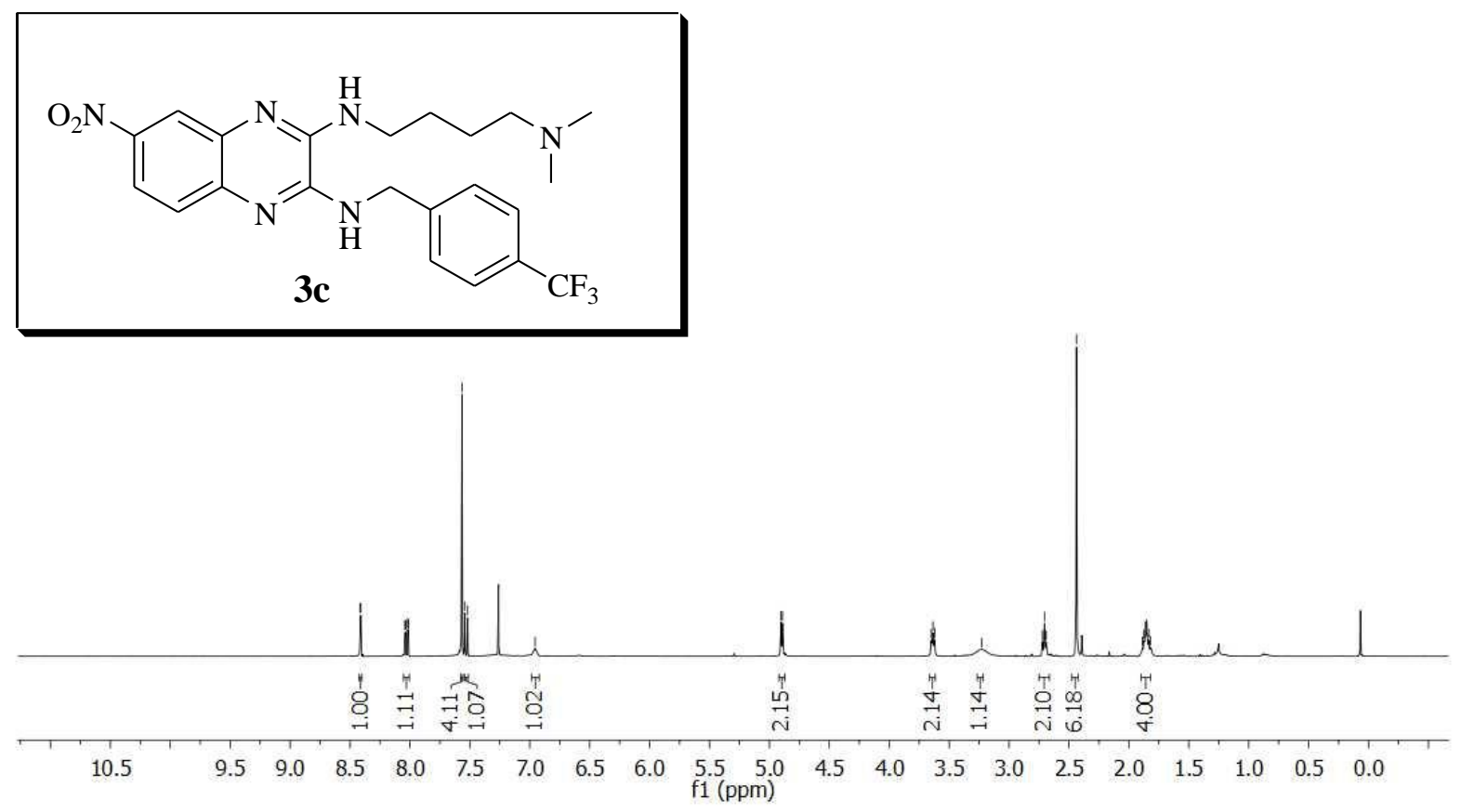

${ }^{13} \mathrm{C}$ NMR of $3 \mathbf{c}\left(\mathrm{CDCl}_{3}, 100 \mathrm{MHz}\right)$ :-

RP-4CF CDA3 400MHz mळ
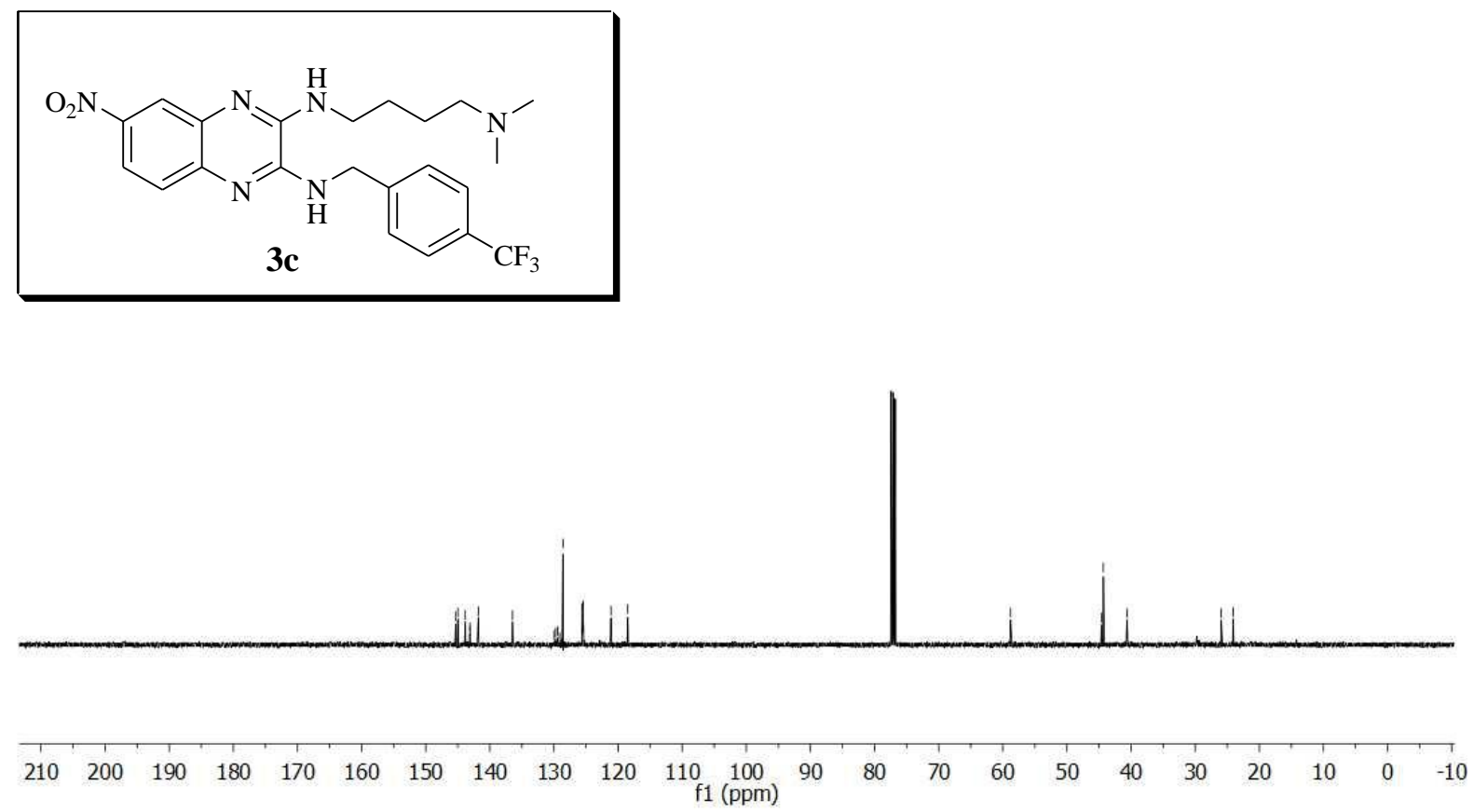
${ }^{1} \mathrm{H}$ NMR of $\mathbf{3 d}\left(\mathrm{CDCl}_{3}, 600 \mathrm{MHz}\right)$ :-

RP-PYCF CDC13 600MHz
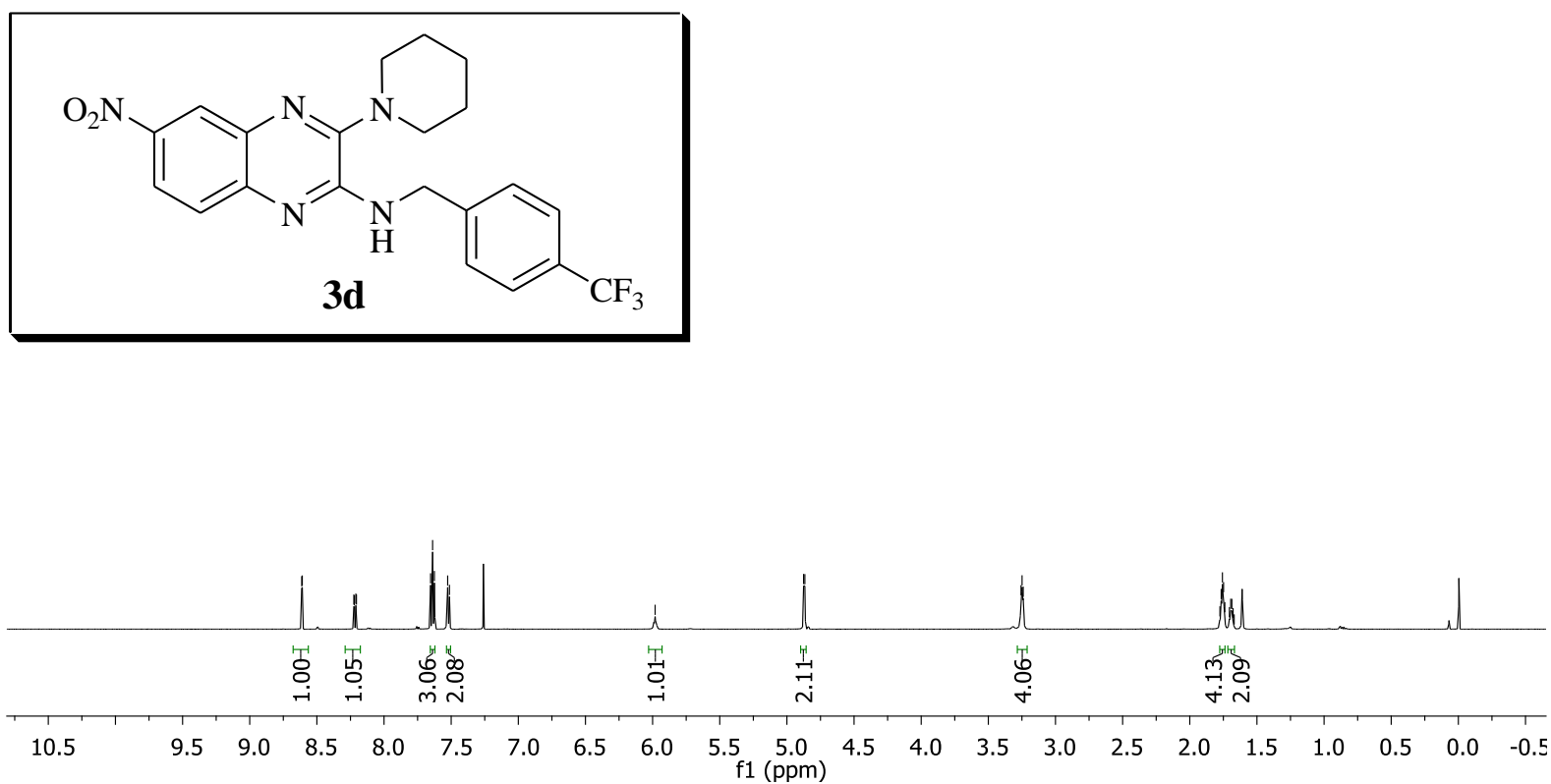

${ }^{13} \mathrm{C} \mathrm{NMR}$ of $\mathbf{3 d}\left(\mathrm{CDCl}_{3}, 150 \mathrm{MHz}\right)$ :-

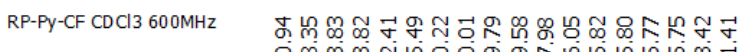

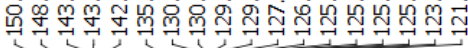

m
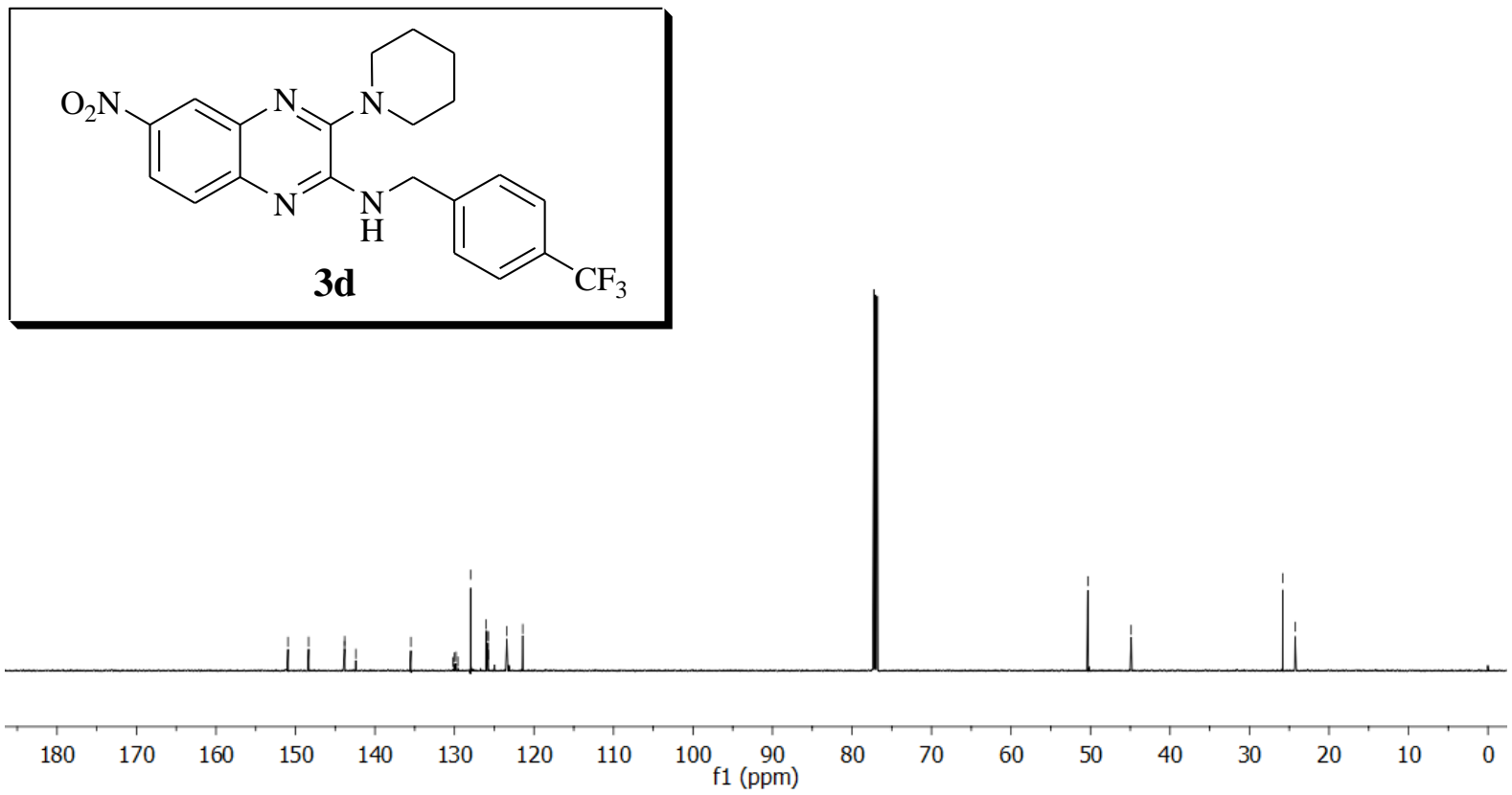
${ }^{1} \mathrm{H}$ NMR of $3 \mathbf{e}\left(\mathrm{CDCl}_{3}, 600 \mathrm{MHz}\right)$ :-

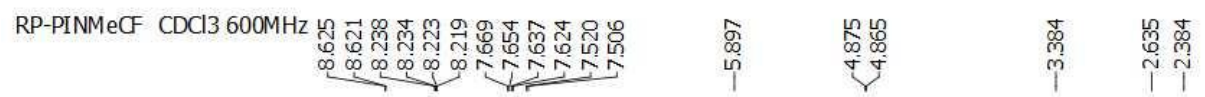
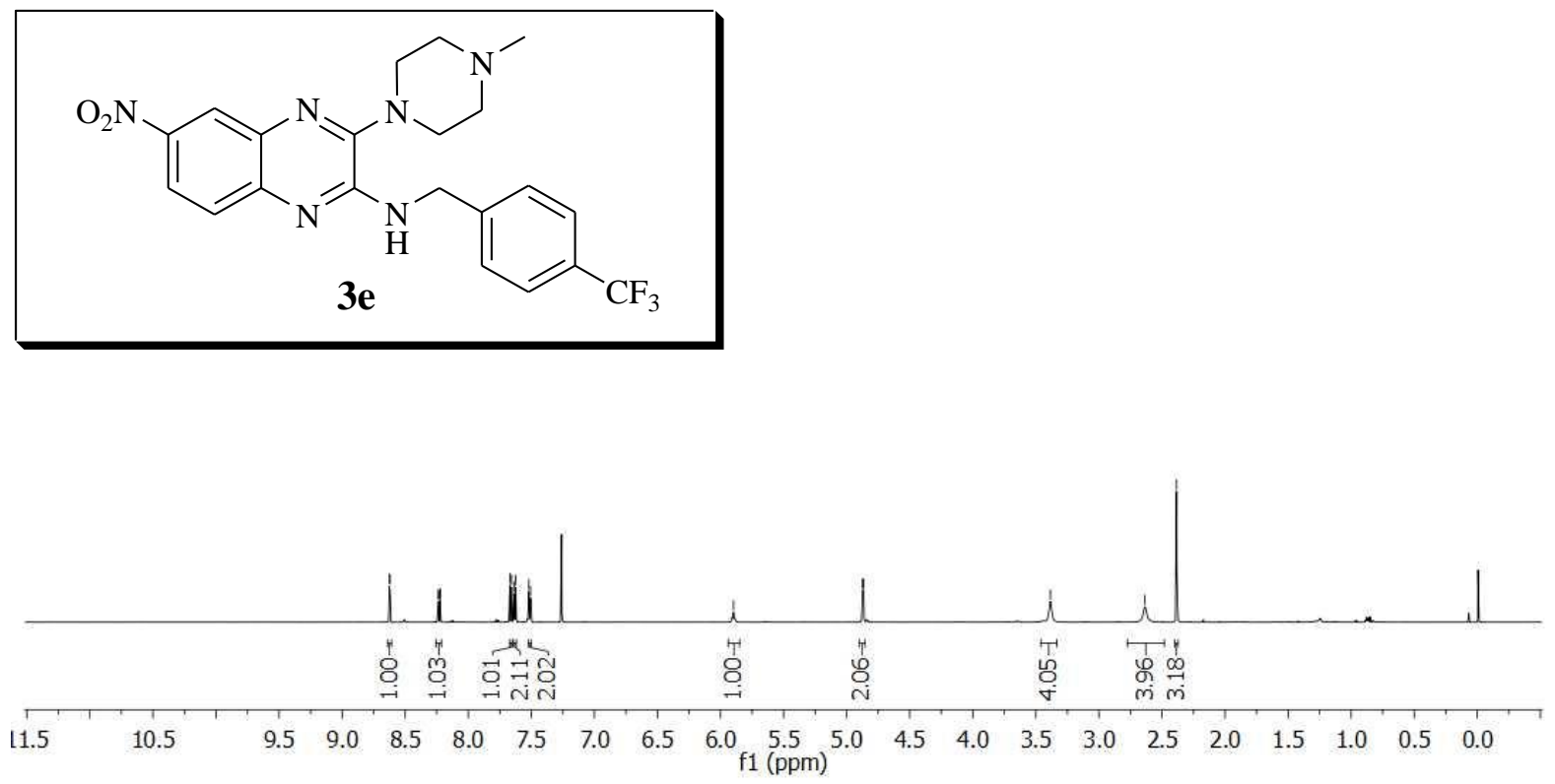

${ }^{13} \mathrm{C}$ NMR of $3 \mathbf{e}\left(\mathrm{CDCl}_{3}, 150 \mathrm{MHz}\right)$ :-

RP-PINMe-CF CDCl3 $600 \mathrm{MHz}$ ๓

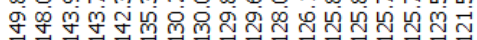

유 움 辡安导
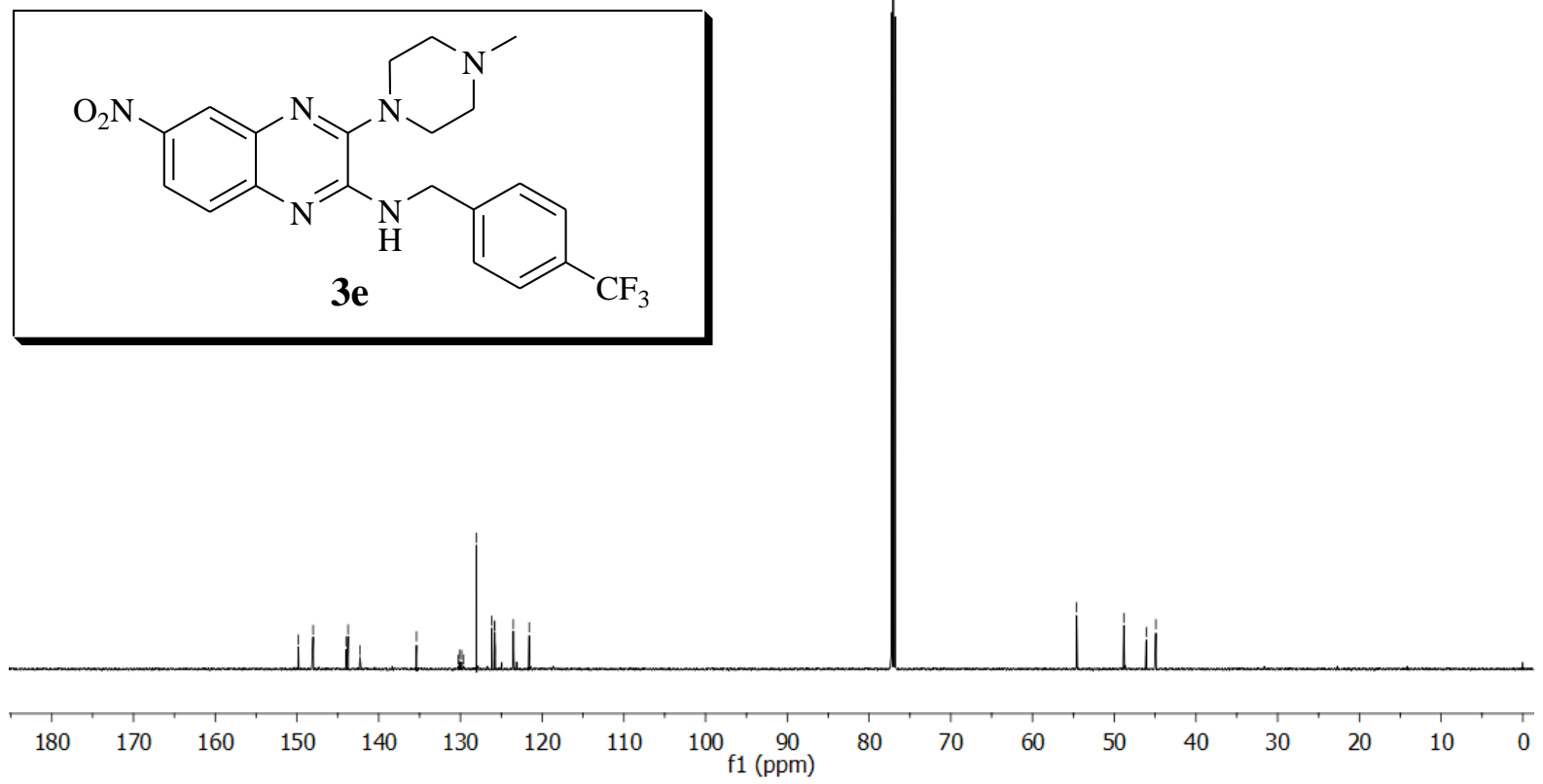
${ }^{1} \mathrm{H}$ NMR of $\mathbf{3 f}\left(\mathrm{CDCl}_{3}, 600 \mathrm{MHz}\right)$ :-

RP-PIN CF CDCl3 $600 \mathrm{MHz}$

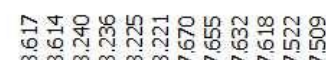

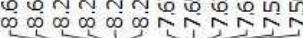

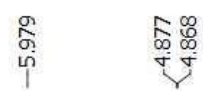

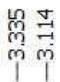
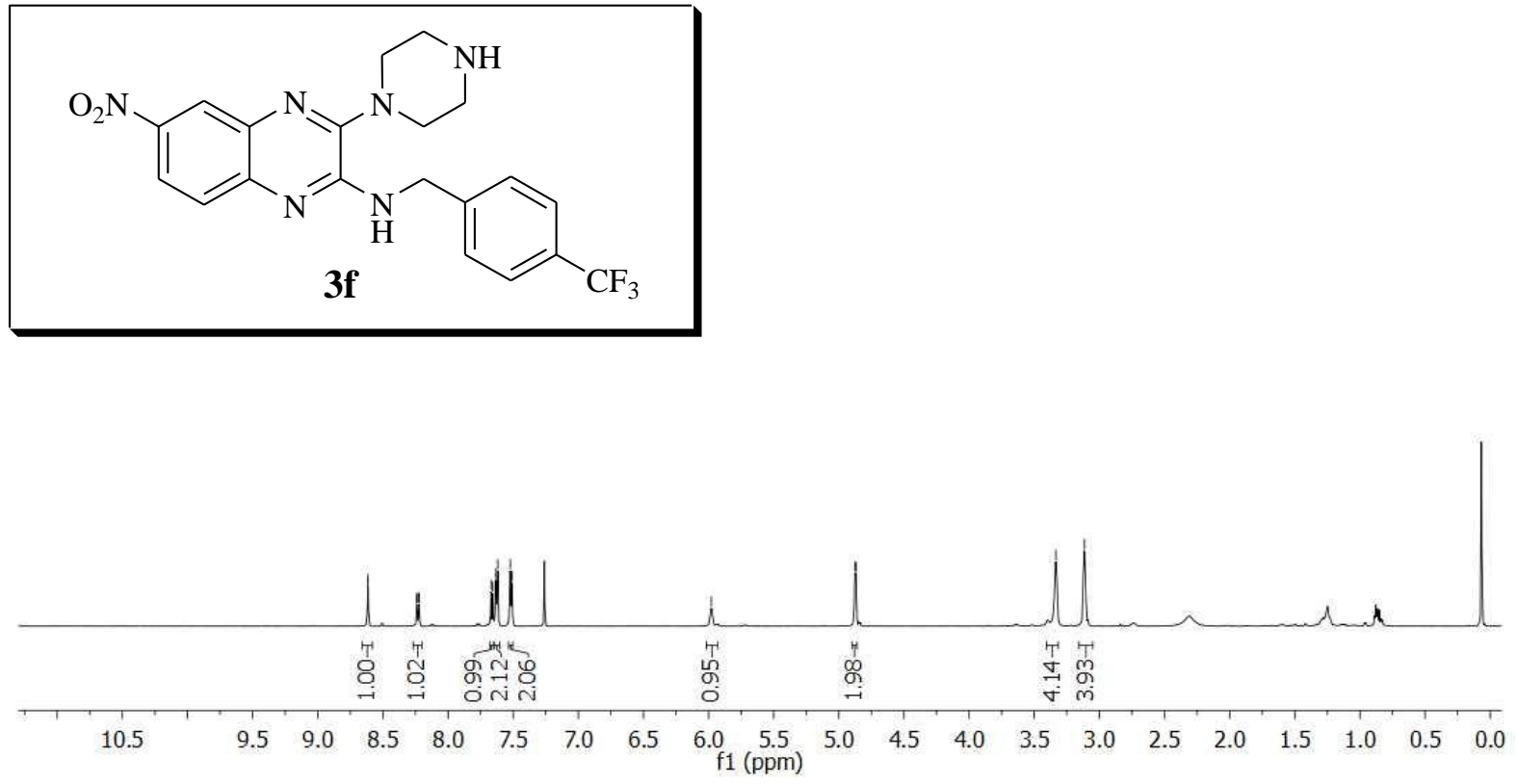

${ }^{13} \mathrm{C}$ NMR of $\mathbf{3 f}\left(\mathrm{CDCl}_{3}, 150 \mathrm{MHz}\right)$ :-

RP-PIN CF CDCl3 $600 \mathrm{MHz}$

궁 \%

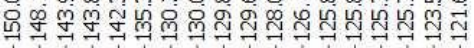
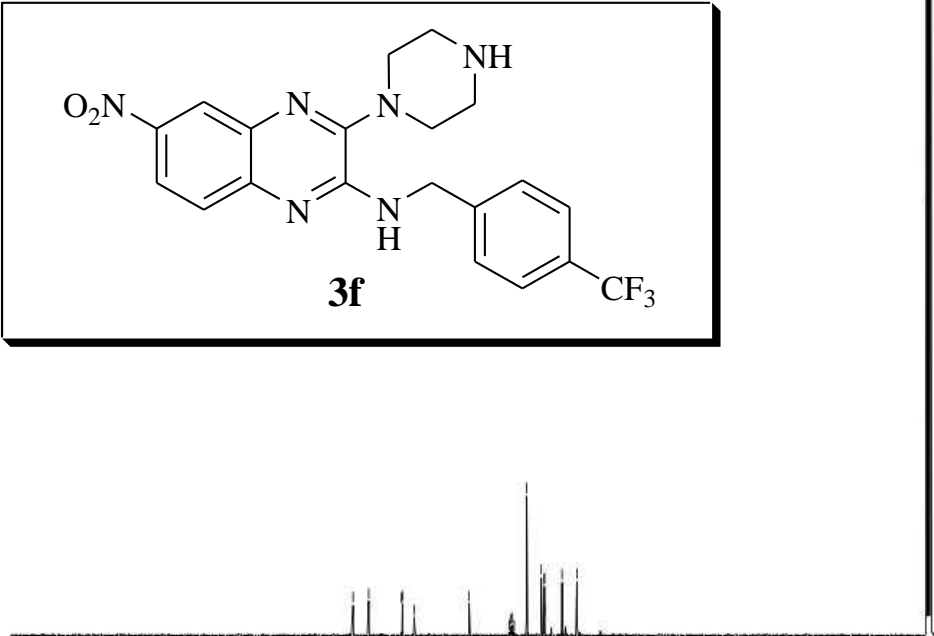
${ }^{1} \mathrm{H}$ NMR of $3 \mathbf{h}\left(\mathrm{CDCl}_{3}, 600 \mathrm{MHz}\right)$ :-

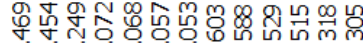

$\underbrace{\infty, 00000000}$

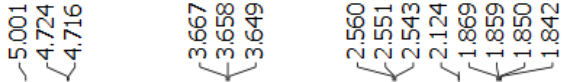

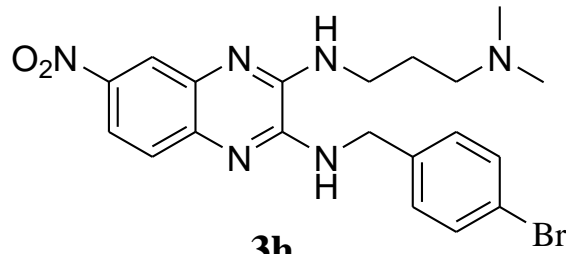

Br

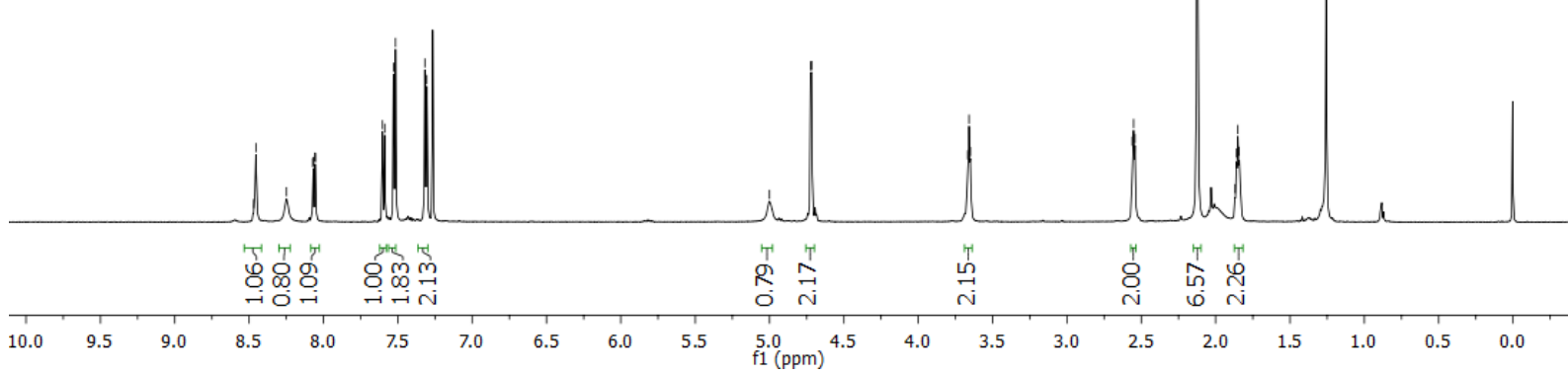

${ }^{13} \mathrm{C}$ NMR of $\mathbf{3 h}\left(\mathrm{CDCl}_{3}, 150 \mathrm{MHz}\right)$ :-

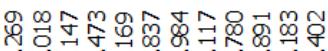

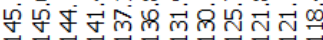<smiles>CN(C)CCCNc1nc2cc([N+](=O)[O-])ccc2nc1NCc1ccc(Br)cc1</smiles>

$\begin{array}{llllllllllll}210 & 200 & 190 & 180 & 170 & 160 & 150 & 140 & 130 & 120 & 110 & \underset{\mathrm{f} 1}{100}(\mathrm{ppm})\end{array}$ 
${ }^{1} \mathrm{H}$ NMR of 3i (DMSO-d $\left.6,600 \mathrm{MHz}\right)$ :-

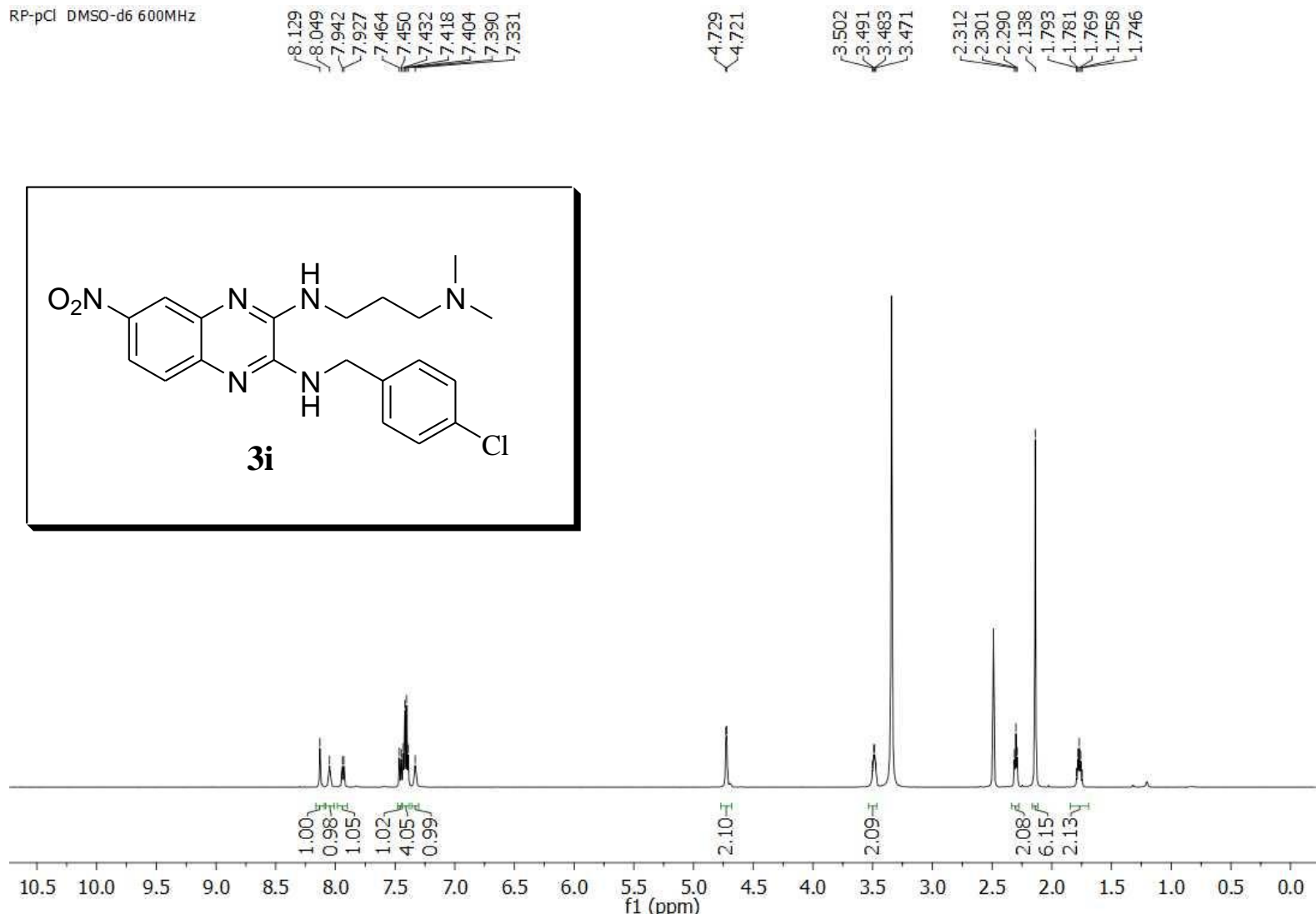

${ }^{13} \mathrm{C}$ NMR of $3 \mathbf{i}\left(\mathrm{DMSO}_{6}, 150 \mathrm{MHz}\right)$ :-
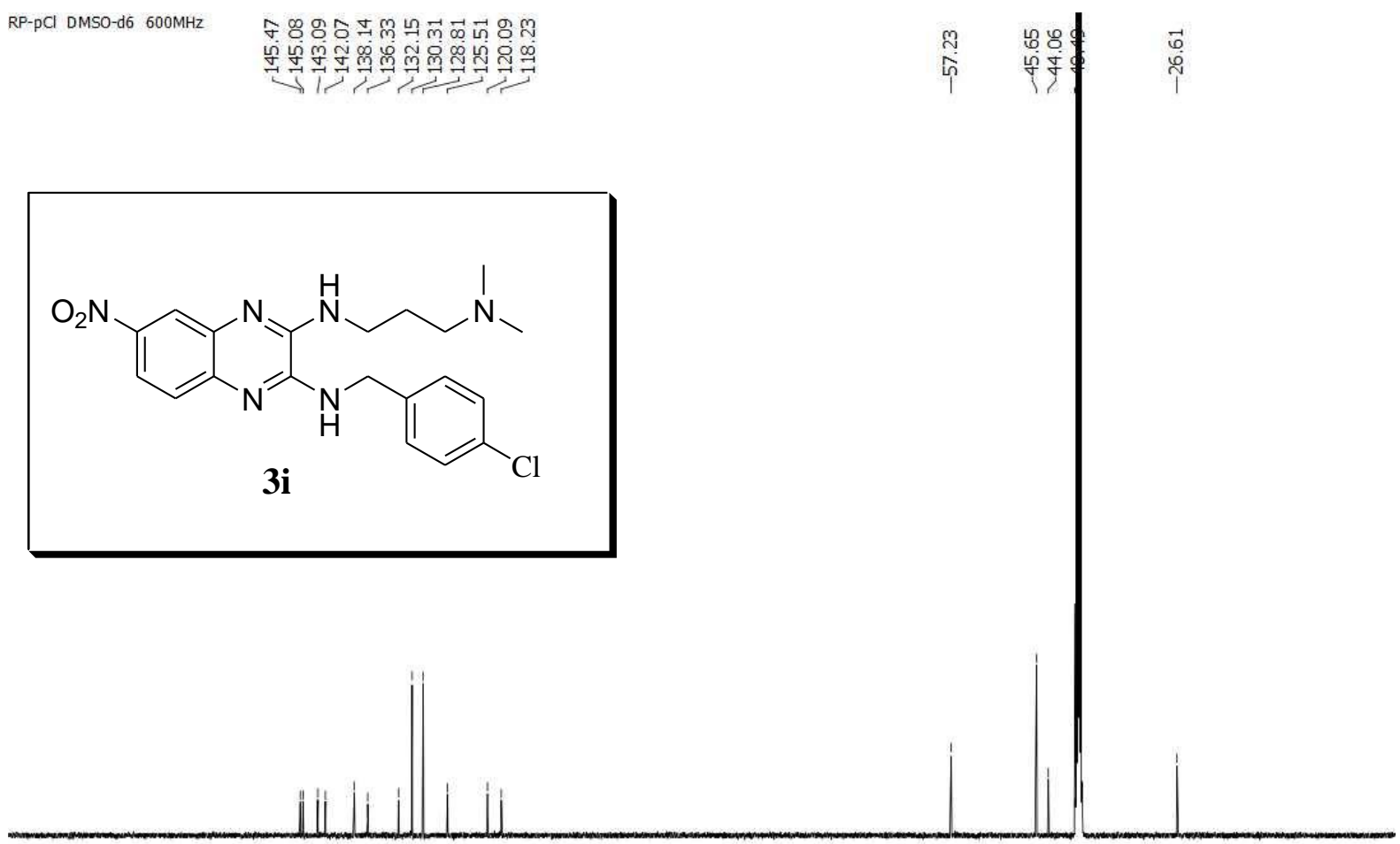

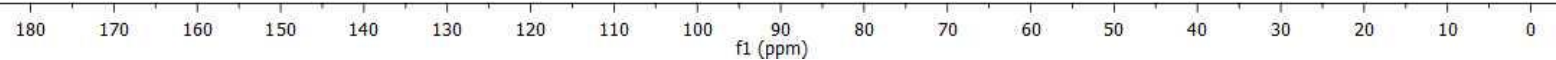


${ }^{1} \mathrm{H}$ NMR of $\mathbf{3 j}\left(\mathrm{CD}_{3} \mathrm{OD}\right.$ in $\left.\mathrm{CDCl}_{3}, 300 \mathrm{MHz}\right)$ :-

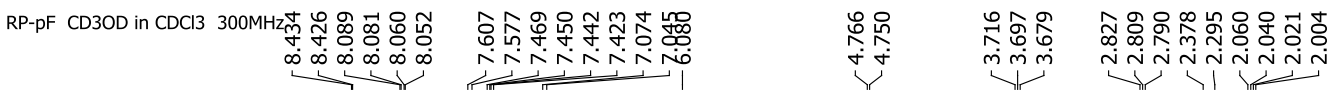
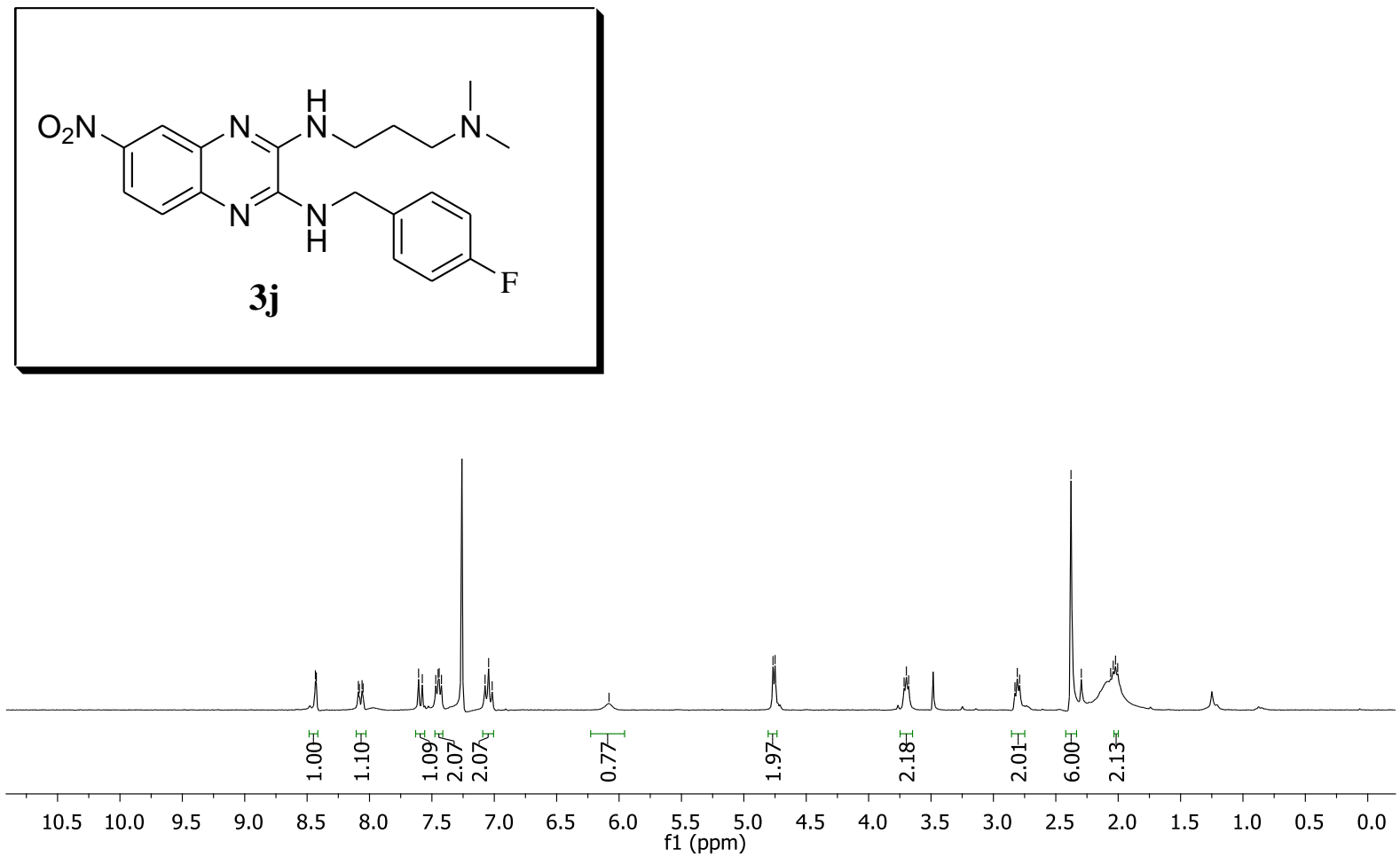

${ }^{13} \mathrm{C}$ NMR of $3 \mathbf{j}\left(\mathrm{DMSO}-\mathrm{d}_{6}, 150 \mathrm{MHz}\right)$ :-

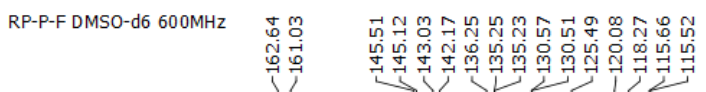
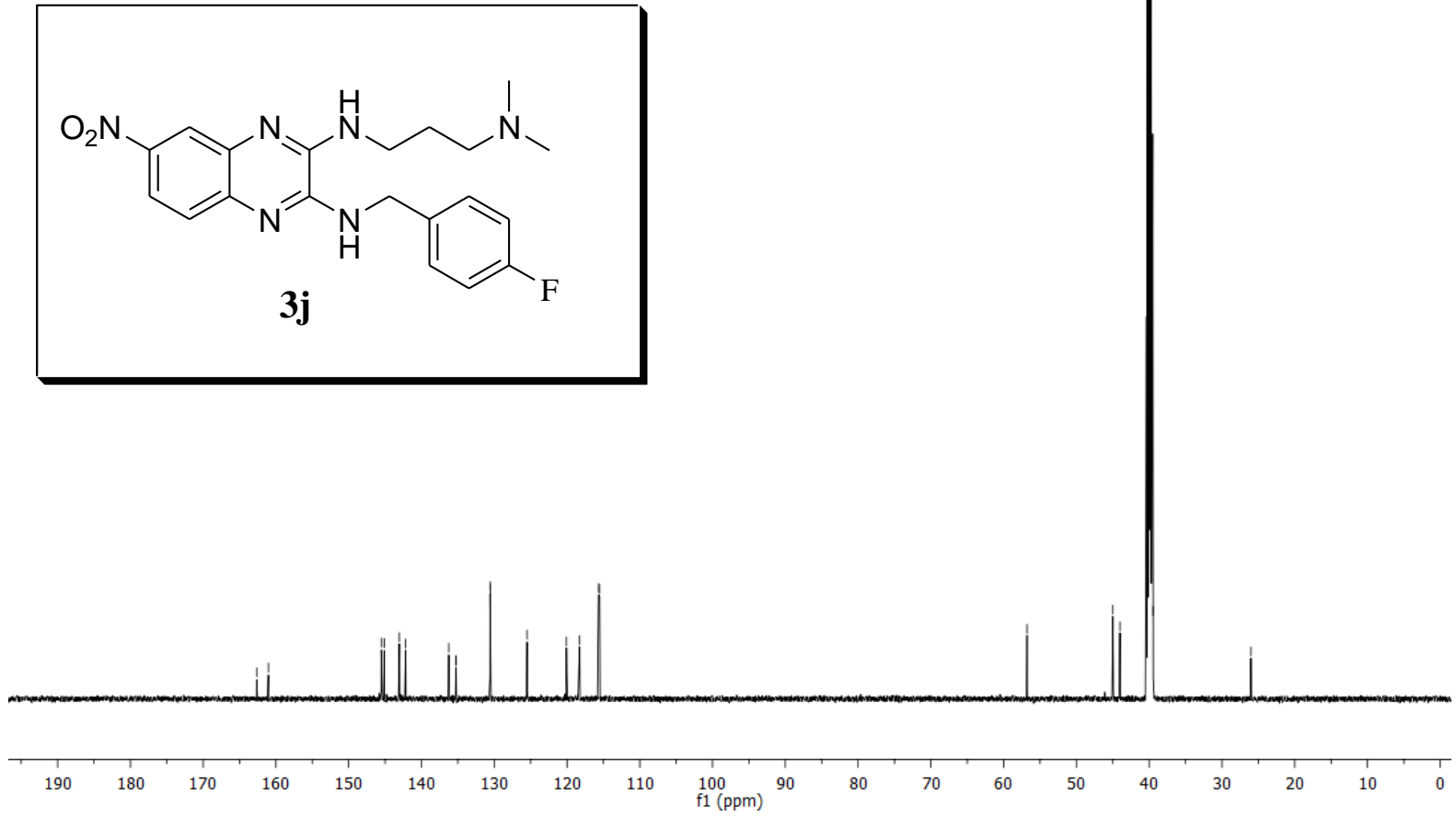
${ }^{1} \mathrm{H}$ NMR of $3 \mathbf{k}\left(\mathrm{CD}_{3} \mathrm{OD}\right.$ in $\left.\mathrm{CDCl}_{3}, 600 \mathrm{MHz}\right)$ :-
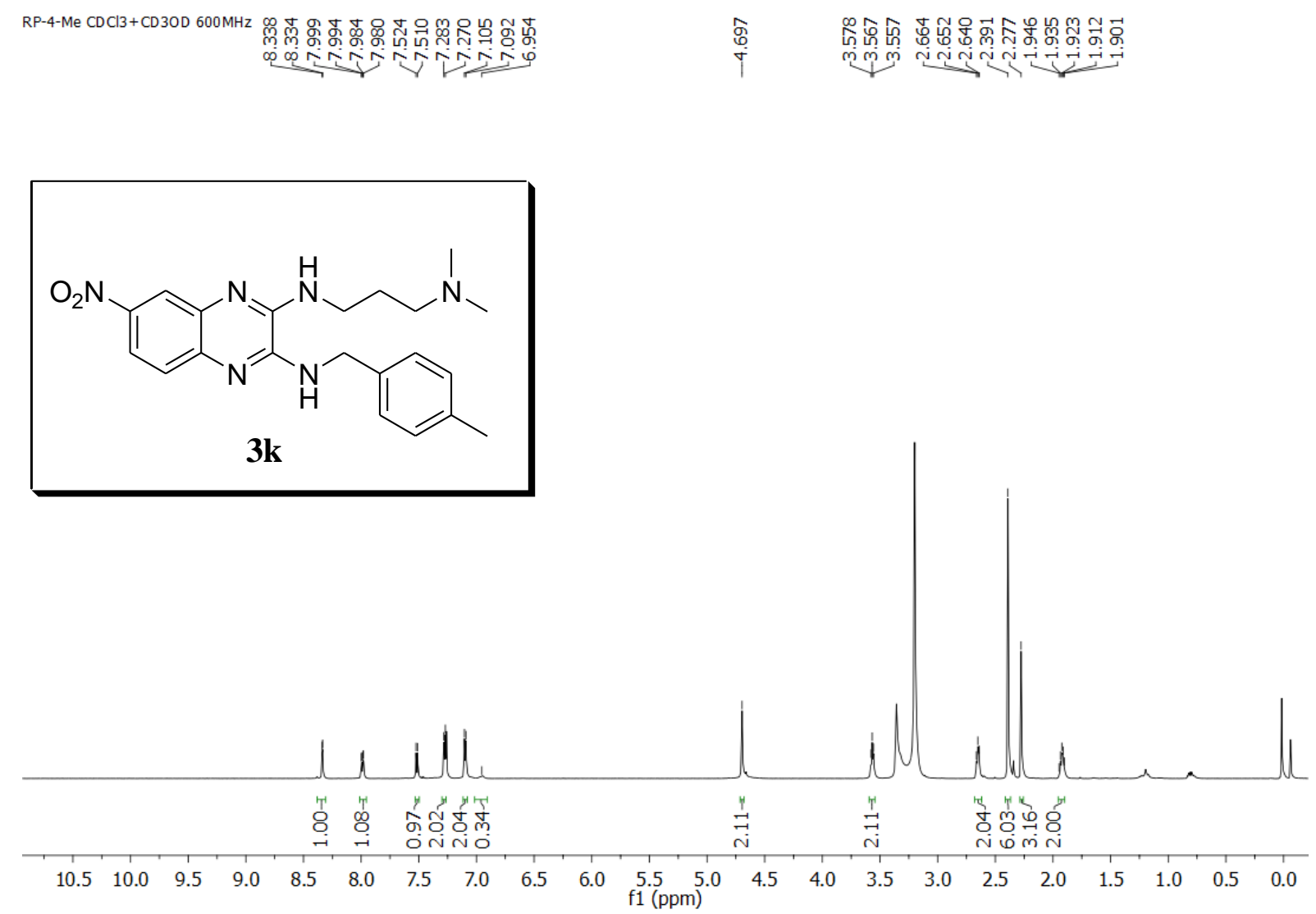

${ }^{13} \mathrm{C}$ NMR of 3k $\left(\mathrm{CD}_{3} \mathrm{OD}\right.$ in $\left.\mathrm{CDCl}_{3}, 150 \mathrm{MHz}\right)$ :-
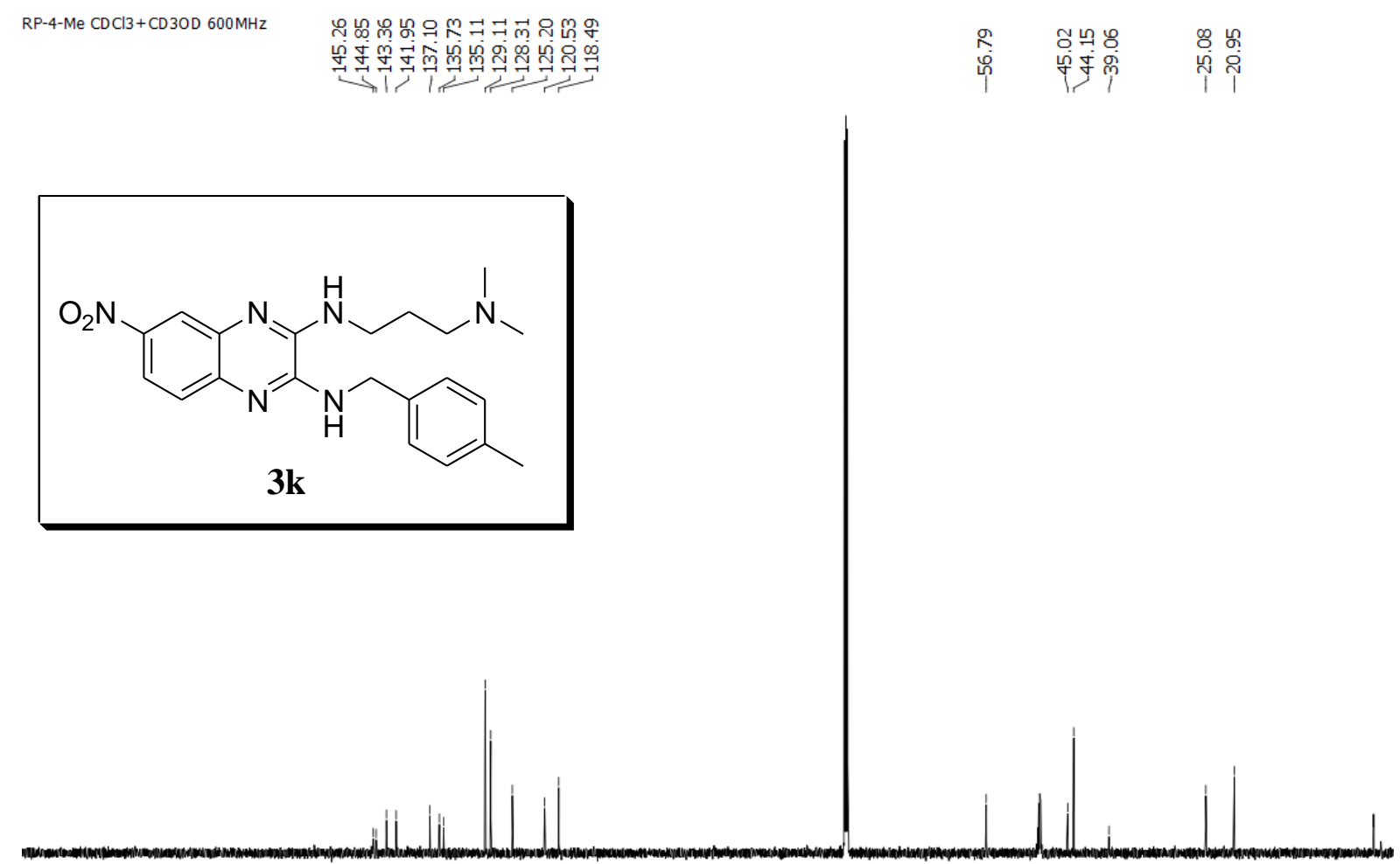

$\begin{array}{llllllllllllllllllll}190 & 180 & 170 & 160 & 150 & 140 & 130 & 120 & 110 & \underset{f 1}{100}(\mathrm{ppm}) & 90 & 80 & 70 & 60 & 50 & 40 & 30 & 20 & 10 & 0\end{array}$ 
${ }^{1} \mathrm{H}$ NMR of $31\left(\mathrm{CD}_{3} \mathrm{OD}\right.$ in $\left.\mathrm{CDCl}_{3}, 600 \mathrm{MHz}\right)$ :-

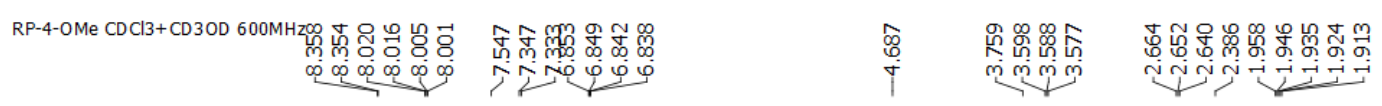
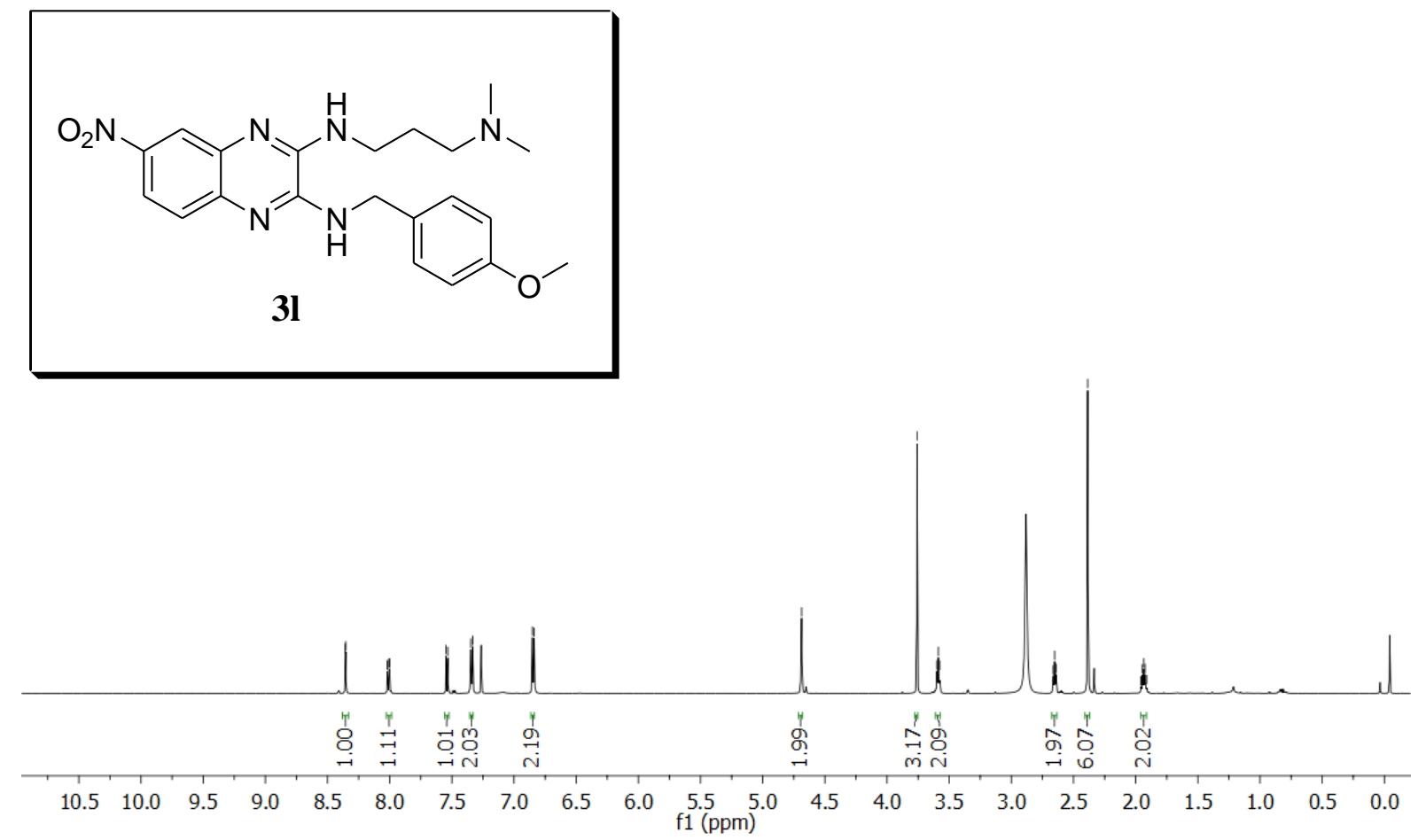

${ }^{13} \mathrm{C}$ NMR of $31\left(\mathrm{CD}_{3} \mathrm{OD}\right.$ in $\left.\mathrm{CDCl}_{3}, 150 \mathrm{MHz}\right)$ :-

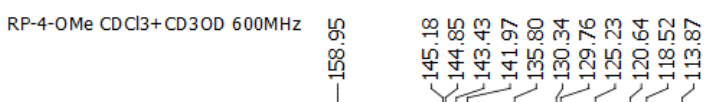

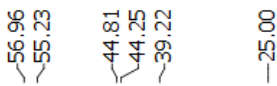
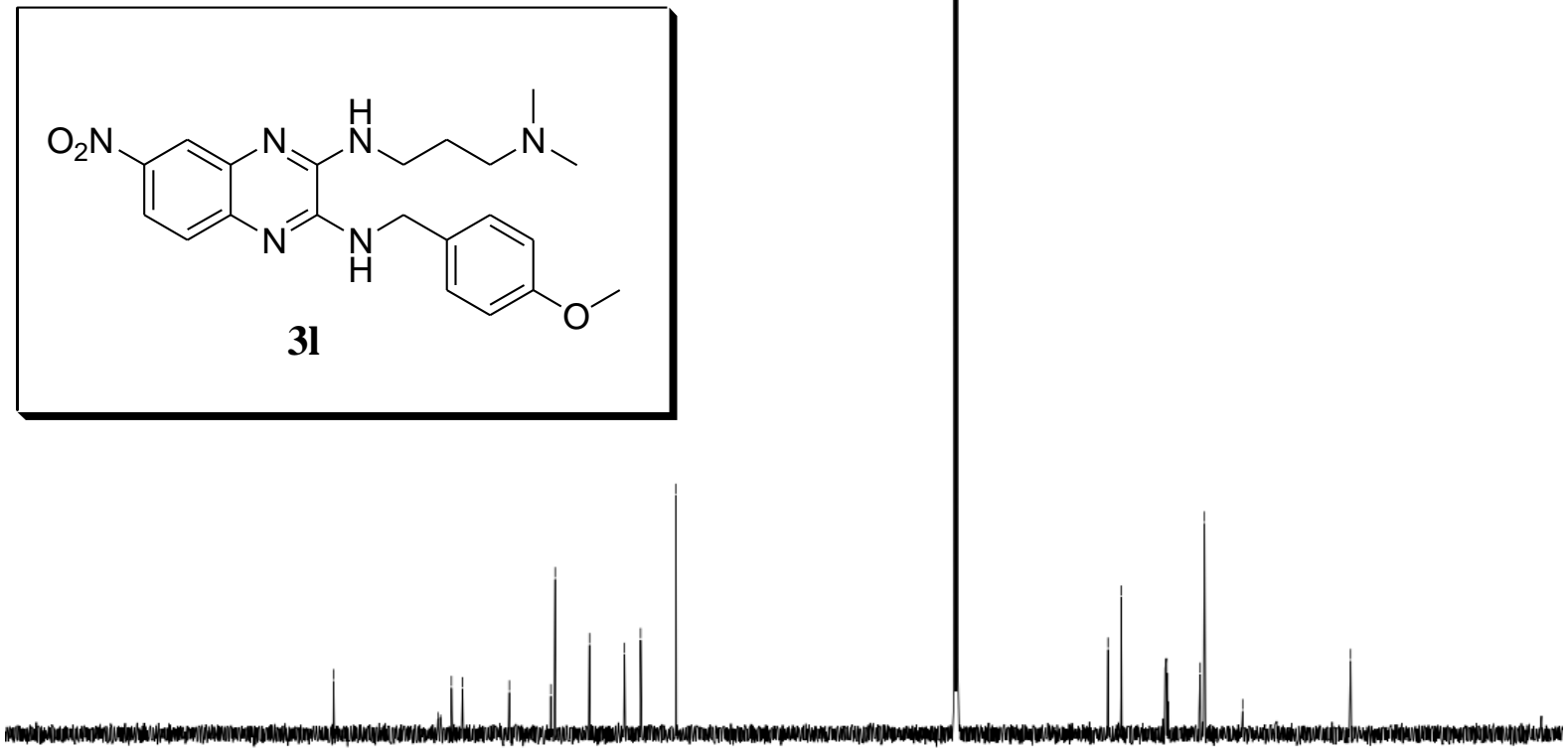

$\begin{array}{lllllllllllllllllllllllll}200 & 190 & 180 & 170 & 160 & 150 & 140 & 130 & 120 & 110 & \begin{array}{c}100 \\ \mathrm{f} 1(\mathrm{ppm})\end{array} & 90 & 80 & 70 & 60 & 50 & 40 & 30 & 20 & 10 & 0\end{array}$ 
${ }^{1} \mathrm{H}$ NMR of $3 \mathbf{m}\left(\mathrm{CD}_{3} \mathrm{OD}\right.$ in $\left.\mathrm{CDCl}_{3}, 600 \mathrm{MHz}\right)$ :-

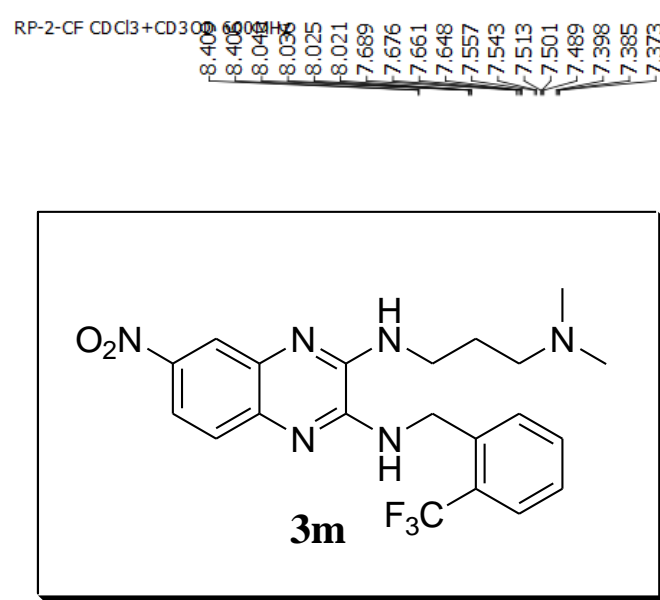

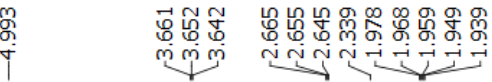

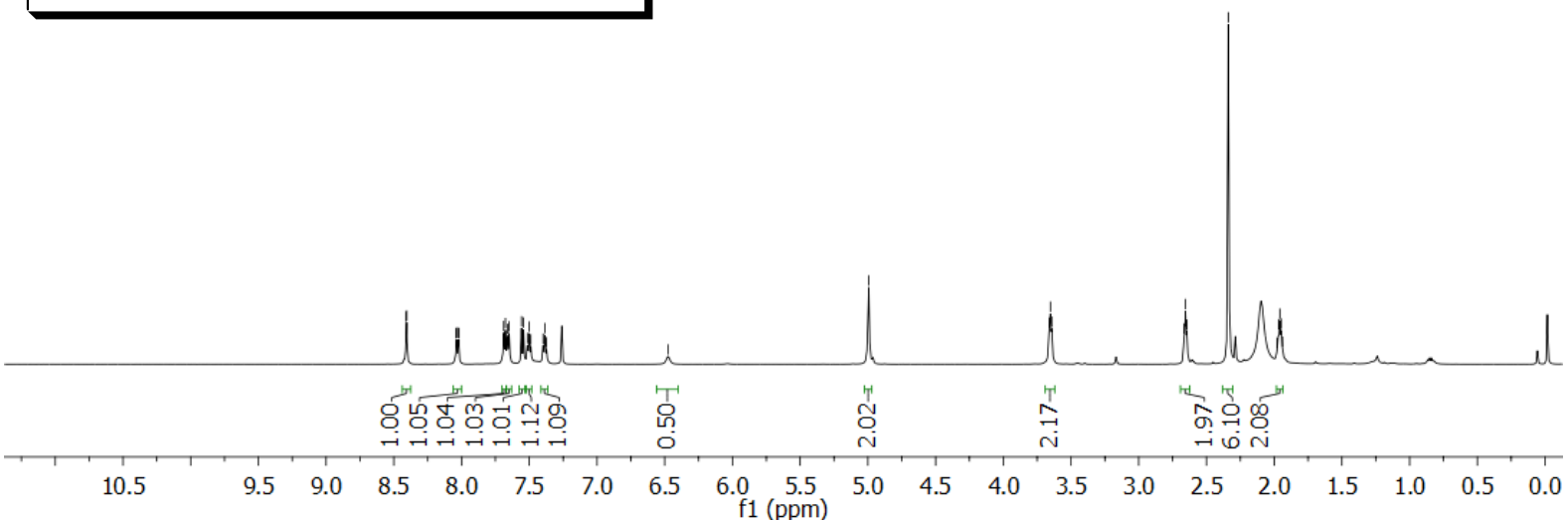

${ }^{13} \mathrm{C}$ NMR of $3 \mathbf{m}\left(\mathrm{CD}_{3} \mathrm{OD}\right.$ in $\left.\mathrm{CDCl}_{3}, 100 \mathrm{MHz}\right)$ :-

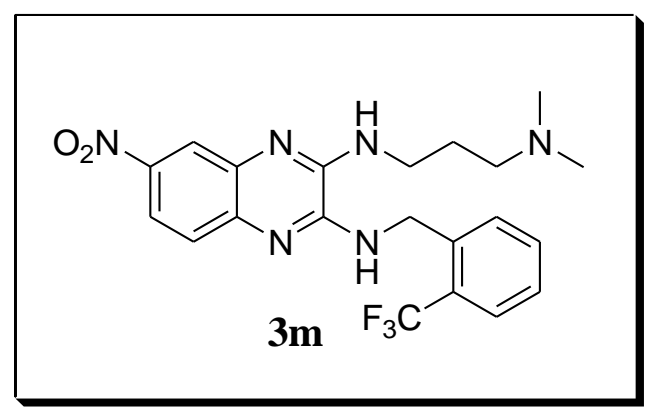

200

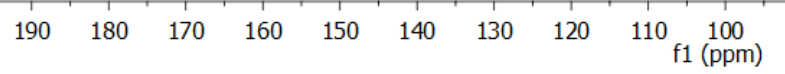


${ }^{1} \mathrm{H}$ NMR of $3 n\left(\mathrm{CDCl}_{3}, 400 \mathrm{MHz}\right)$ :-

RP-2-Br CCI3 400MHz
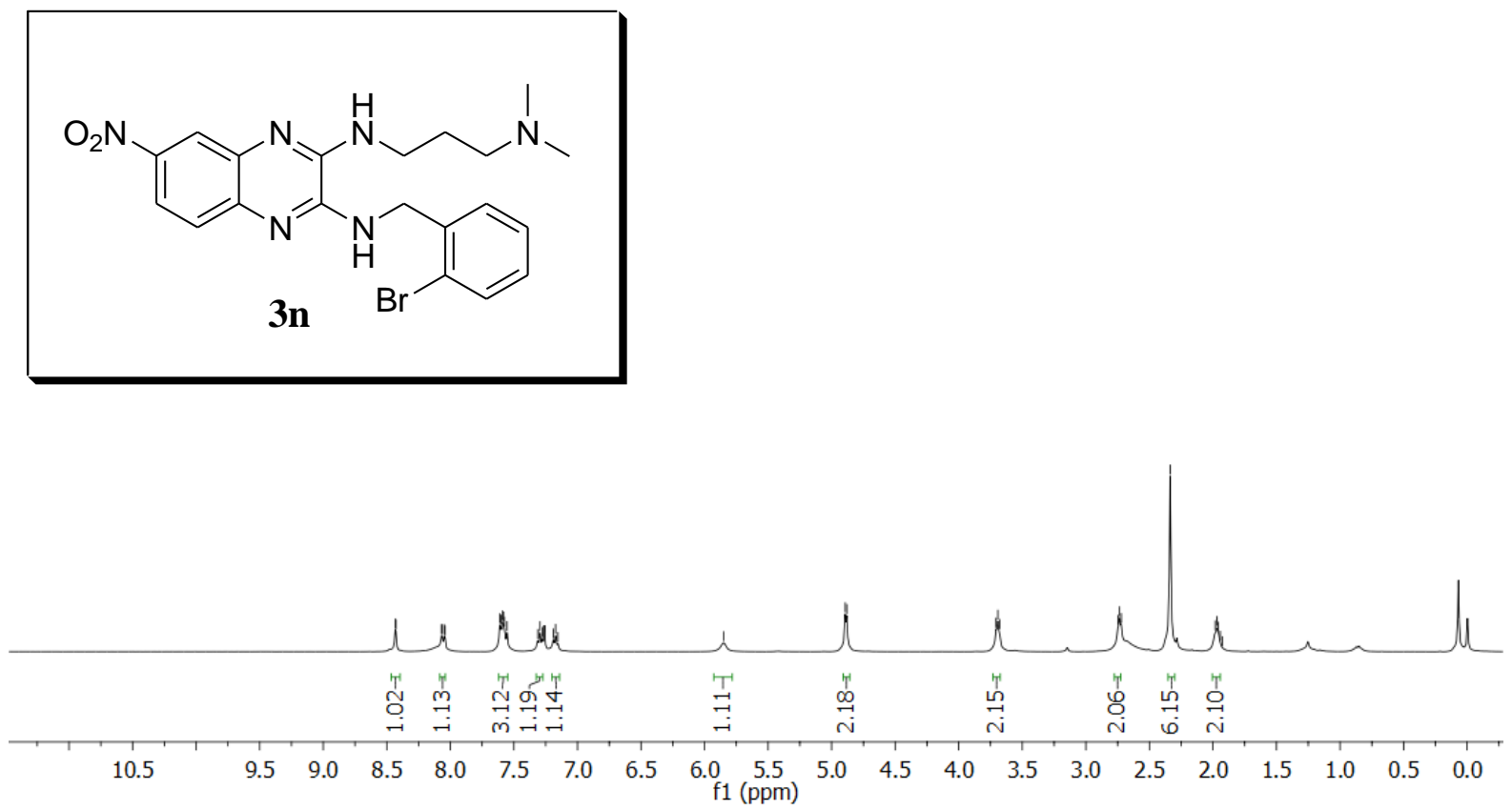

${ }^{13} \mathrm{C}$ NMR of 3n $\left(\mathrm{CDCl}_{3}, 150 \mathrm{MHz}\right)$ :-

$\mathrm{RP}-2-\mathrm{Br} \mathrm{CDCl} 3600 \mathrm{MHz}$

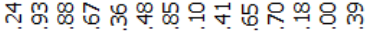

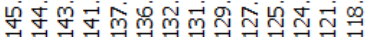

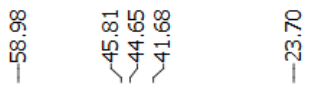
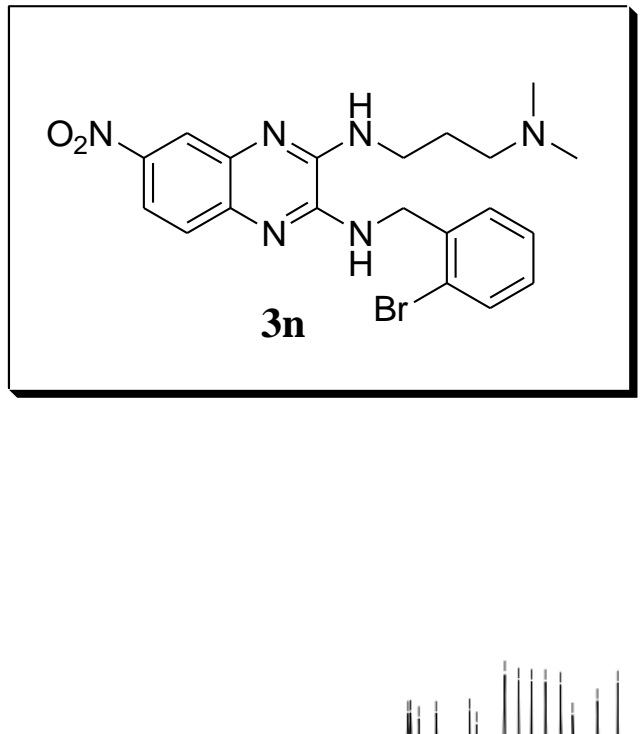

190

$\begin{array}{lll}180 & 170 \quad 160\end{array}$

$150 \quad 140 \quad 130$

$\begin{array}{llc}120 & 110 & 100 \\ \mathrm{f} 1(\mathrm{ppm})\end{array} 90$ 
${ }^{1} \mathrm{H}$ NMR of $3 o\left(\mathrm{CD}_{3} \mathrm{OD}\right.$ in $\left.\mathrm{CDCl}_{3}, 600 \mathrm{MHz}\right)$ :-

RP-2-Me CDCl3+CO300 600MHz
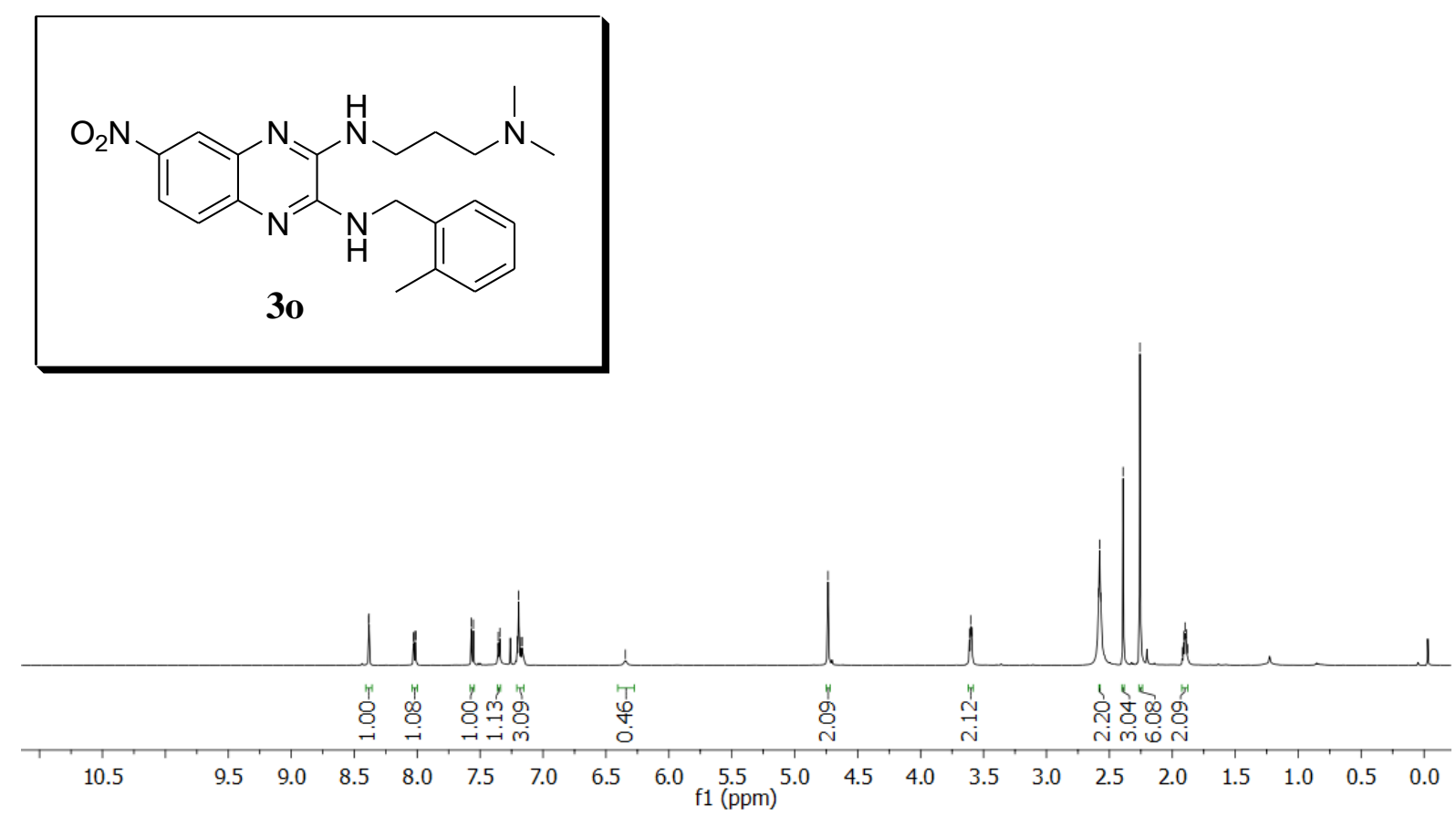

${ }^{13} \mathrm{C}$ NMR of $30\left(\mathrm{CD}_{3} \mathrm{OD}\right.$ in $\left.\mathrm{CDCl}_{3}, 150 \mathrm{MHz}\right)$ :-

RP-2-Me CDCl3+CD30D 600MHz N

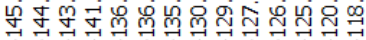

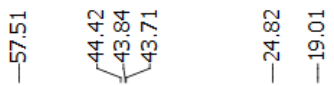
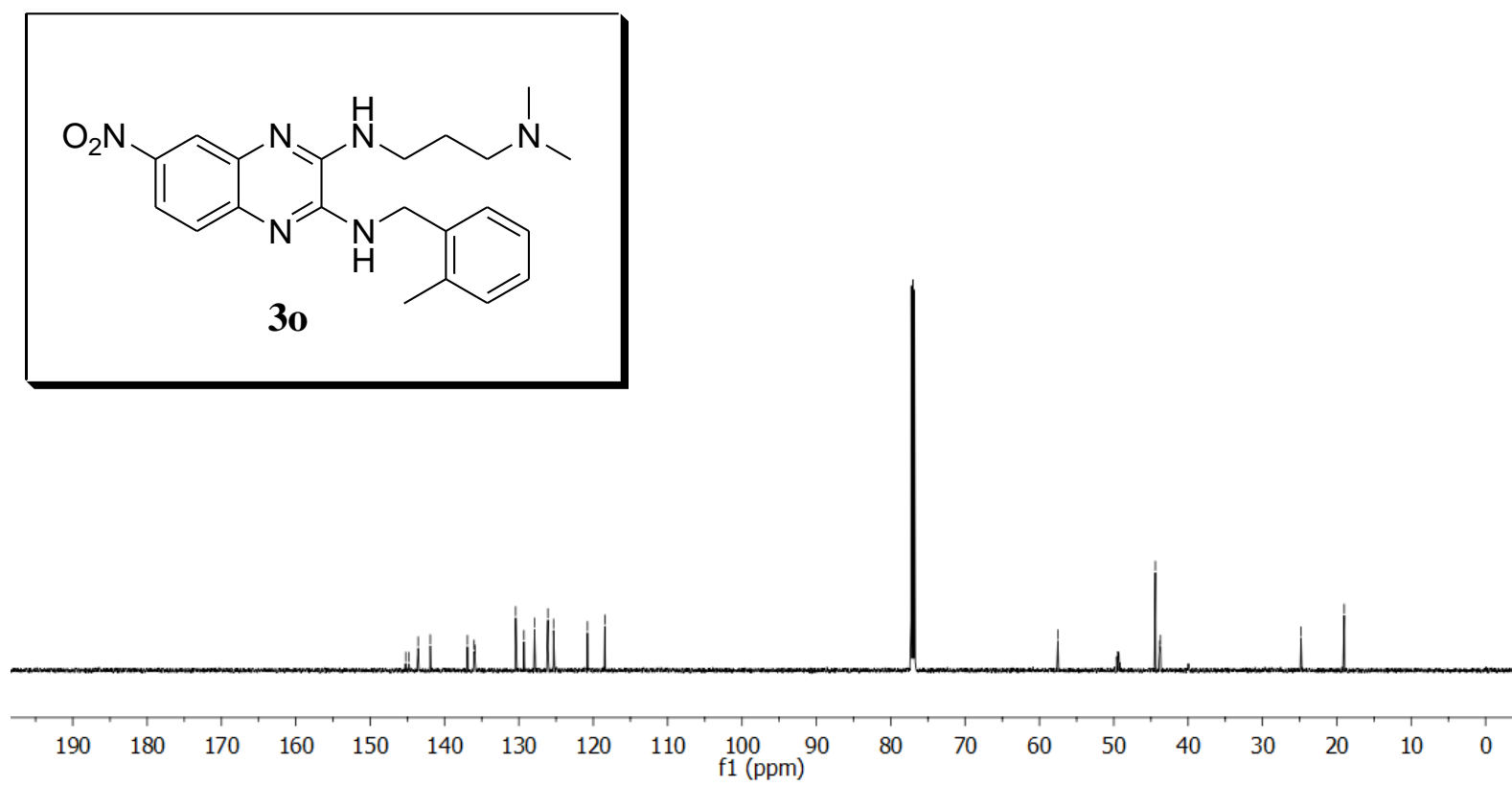
${ }^{1} \mathrm{H}$ NMR of $3 \mathbf{p}\left(\mathrm{CD}_{3} \mathrm{OD}\right.$ in $\left.\mathrm{CDCl}_{3}, 600 \mathrm{MHz}\right)$ :-

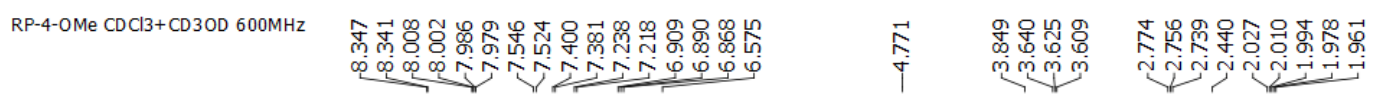

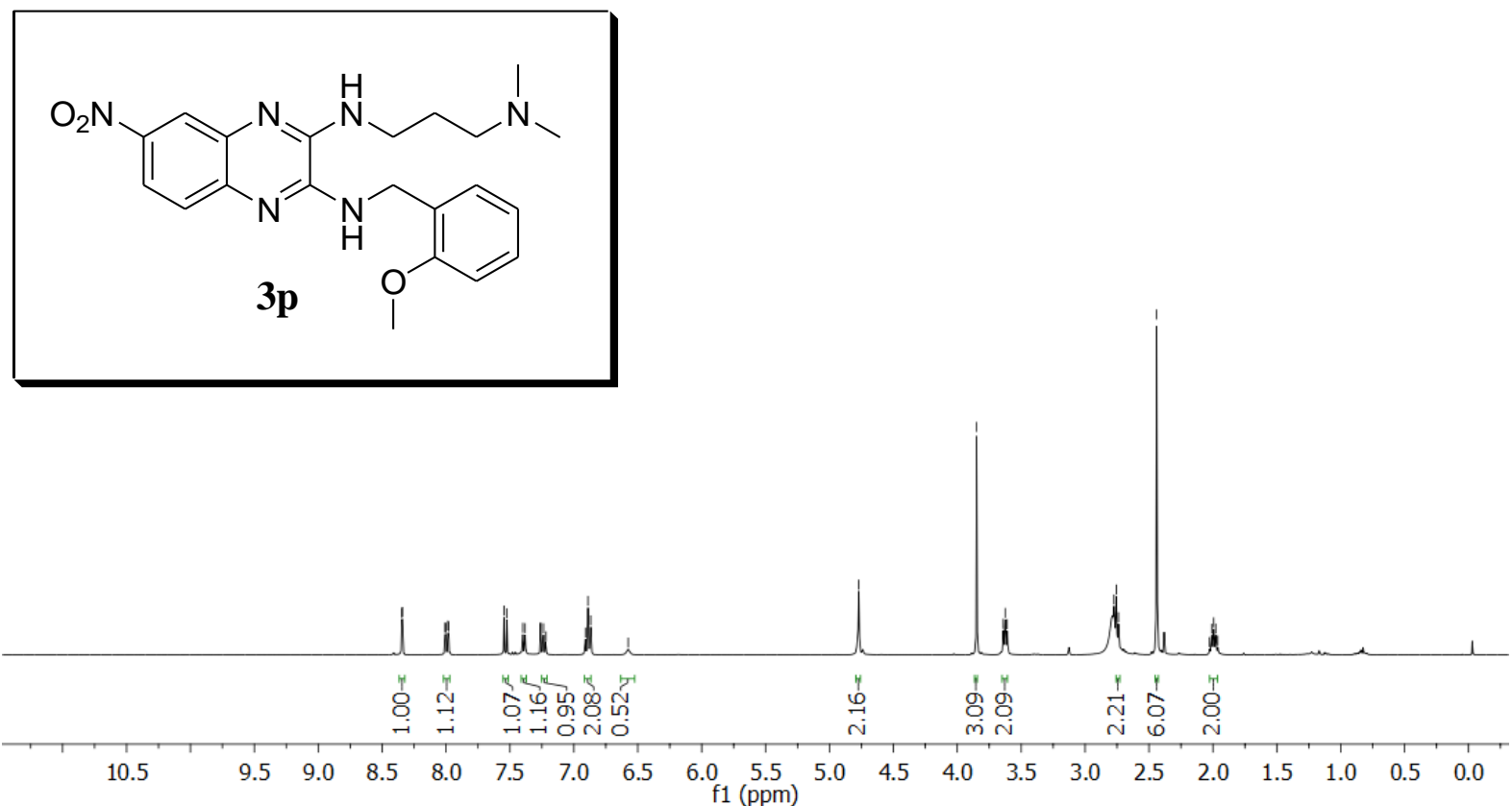

${ }^{13} \mathrm{C}$ NMR of $3 \mathbf{p}\left(\mathrm{CD}_{3} \mathrm{OD}\right.$ in $\left.\mathrm{CDCl}_{3}, 100 \mathrm{MHz}\right)$ :-

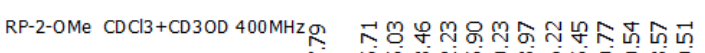

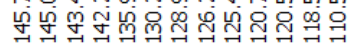

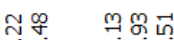

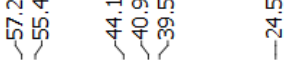
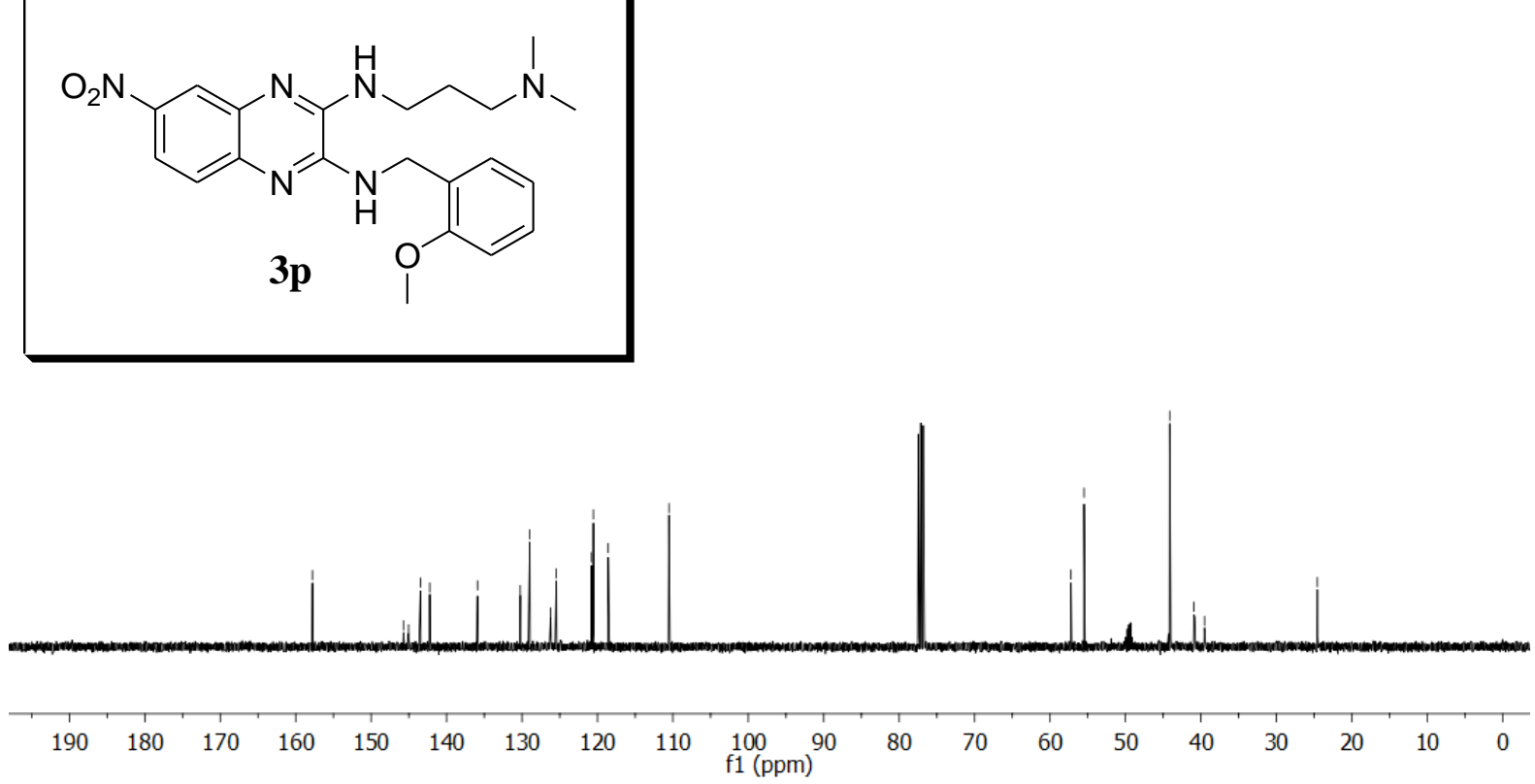
${ }^{1} \mathrm{H}$ NMR of 3q $\left(\mathrm{CDCl}_{3}, 600 \mathrm{MHz}\right)$ :-

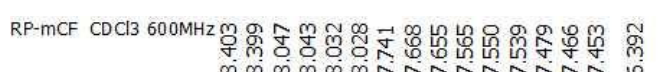

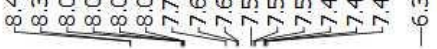

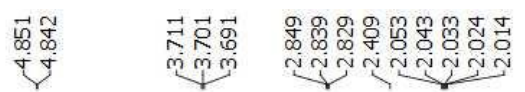

(1)

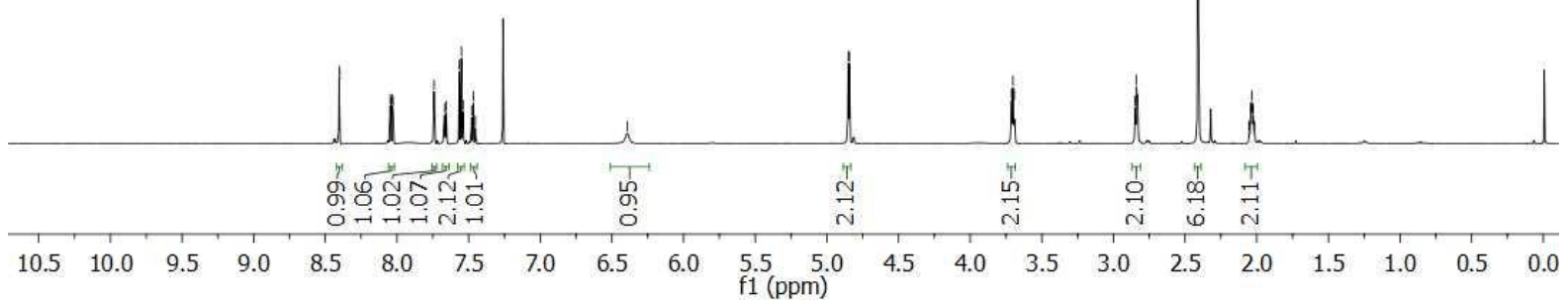

${ }^{13} \mathrm{C}$ NMR of $\mathbf{3 q}\left(\mathrm{CDCl}_{3}, 150 \mathrm{MHz}\right):-$

RP-m-CF CDCl3 6mpmp

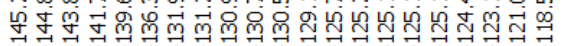

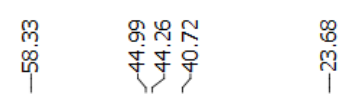

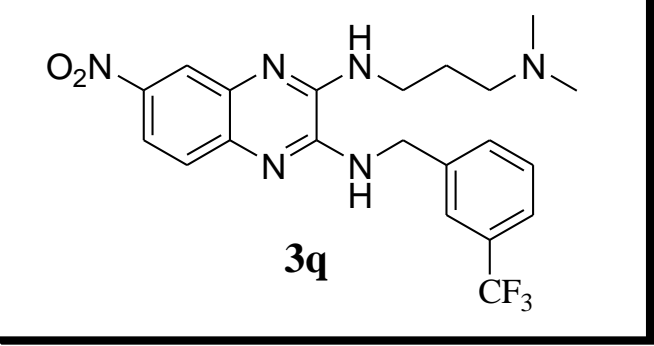

岁

$\begin{array}{llllllllll}80 & 170 & 160 & 150 & 140 & 130 & 120 & 110 & 100 & \underset{\mathrm{f} 1(\mathrm{ppm})}{90}\end{array}$ 
${ }^{1} \mathrm{H}$ NMR of $3 \mathbf{r}\left(\mathrm{CDCl}_{3}, 600 \mathrm{MHz}\right)$ :-

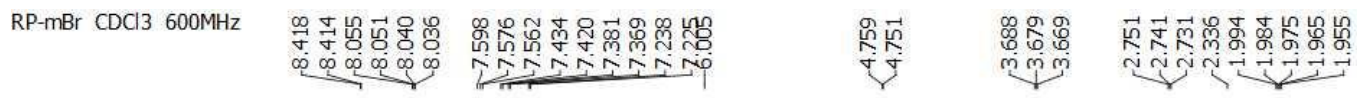
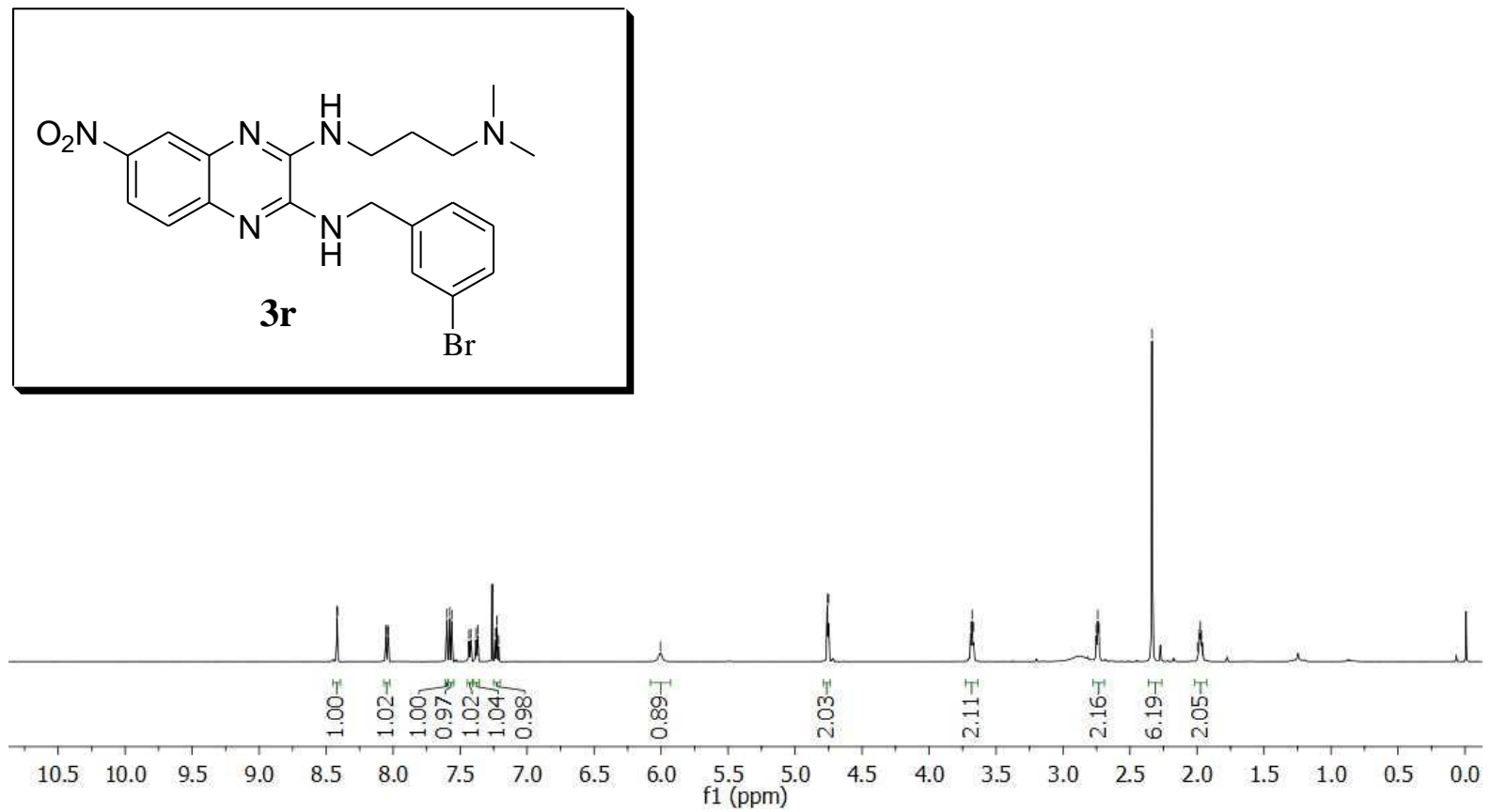

${ }^{13} \mathrm{C}$ NMR of $3 r\left(\mathrm{CDCl}_{3}, 150 \mathrm{MHz}\right)$ :-

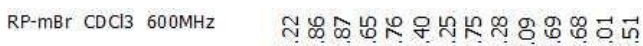

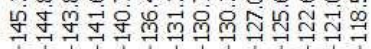

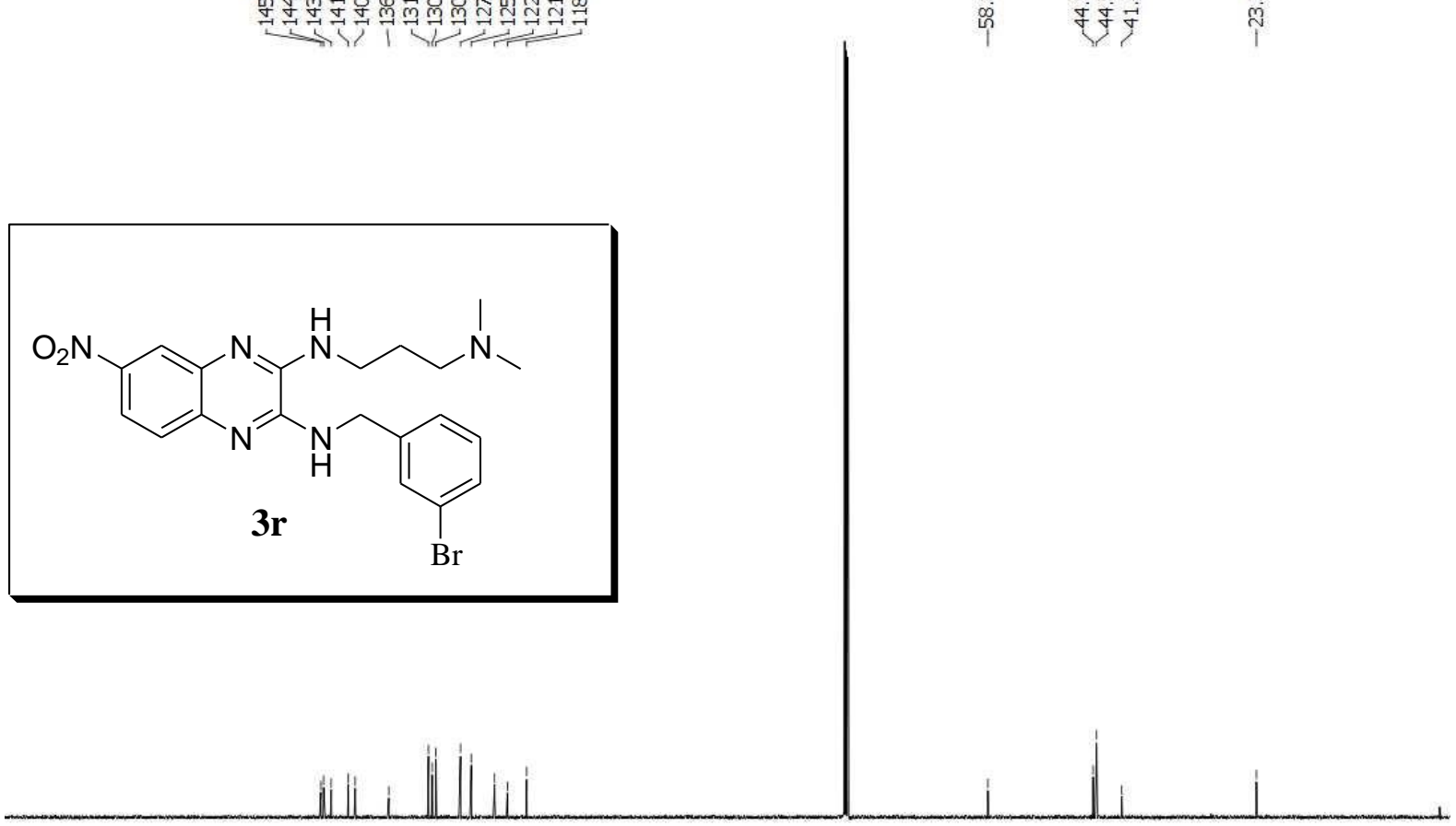

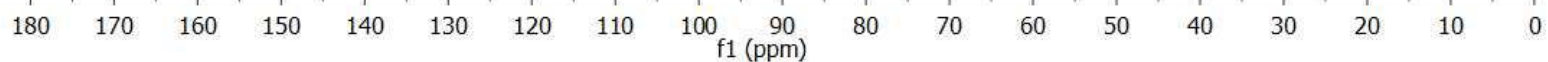


${ }^{1} \mathrm{H}$ NMR of $3 \mathbf{s}\left(\right.$ DMSO- $\left.\mathrm{d}_{6}, 400 \mathrm{MHz}\right)$ :-
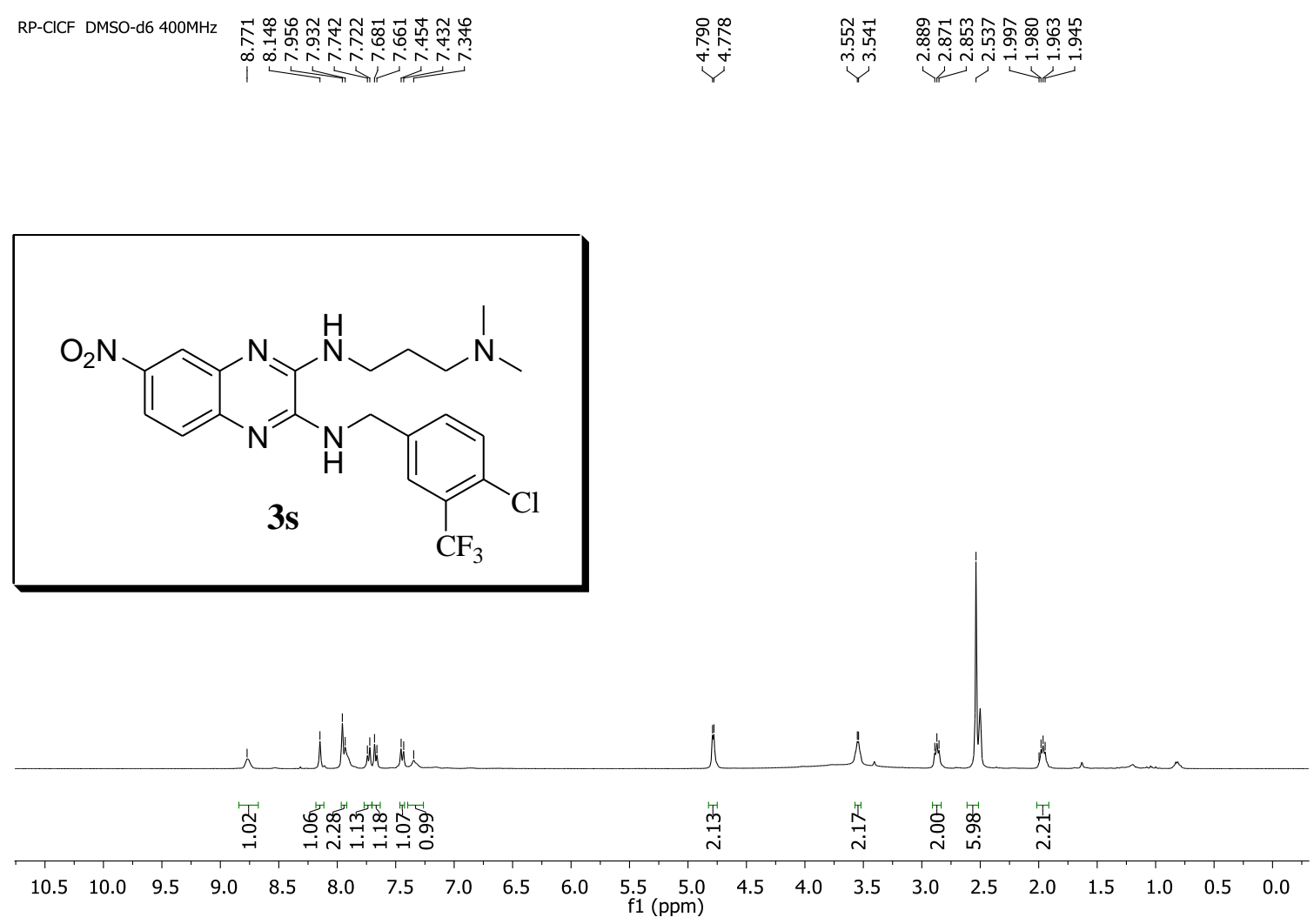

${ }^{13} \mathrm{C}$ NMR of 3s (DMSO-d $\left.6,100 \mathrm{MHz}\right):-$

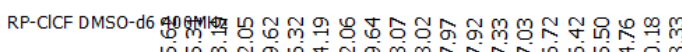

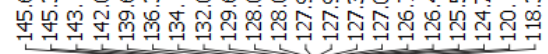

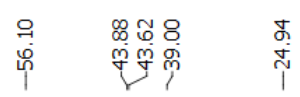

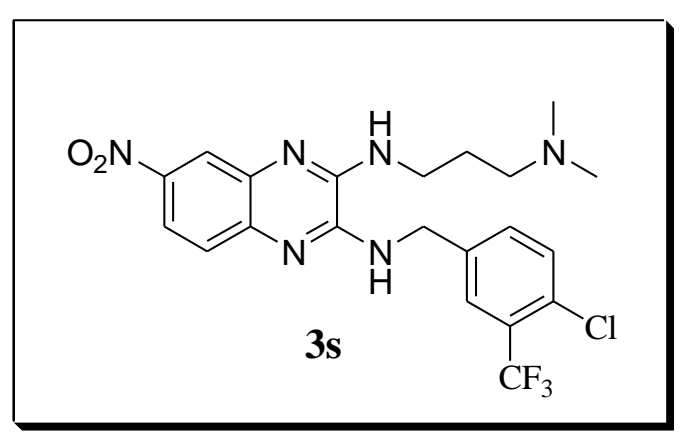

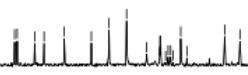

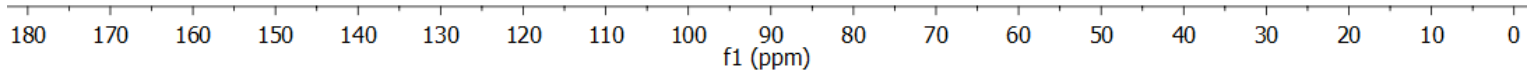


${ }^{1} \mathrm{H}$ NMR of $\mathbf{3 t}\left(\mathrm{CDCl}_{3}, 600 \mathrm{MHz}\right)$ :-

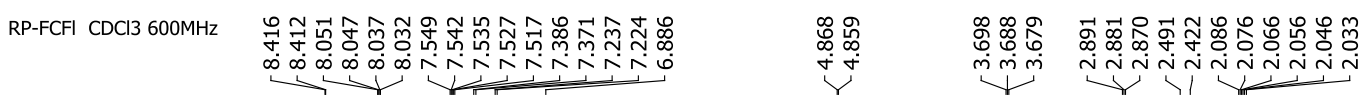
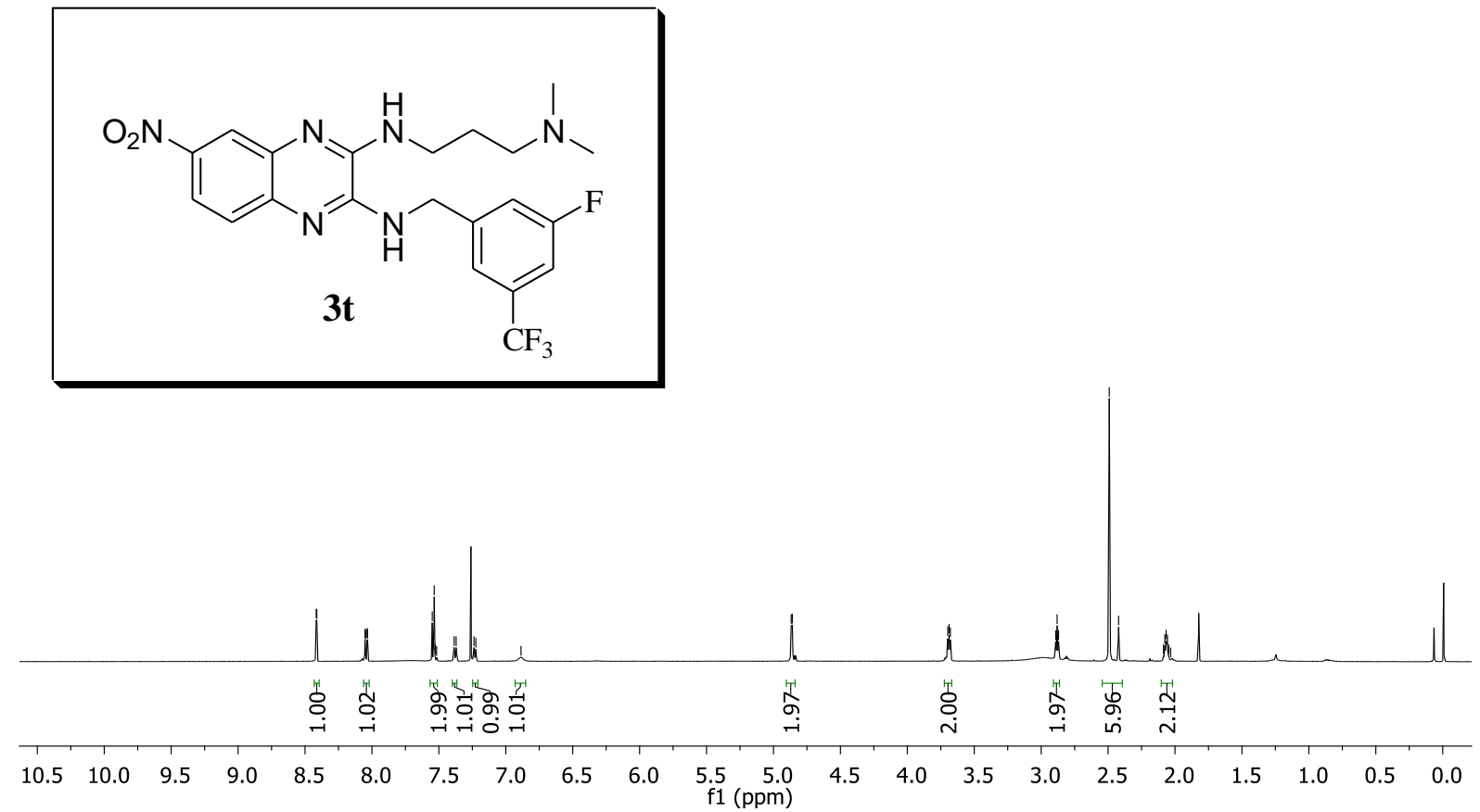

${ }^{13} \mathrm{C}$ NMR of $3 \mathbf{t}\left(\right.$ DMSO- $\left.\mathrm{d}_{6}, 100 \mathrm{MHz}\right)$ :-

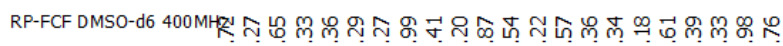

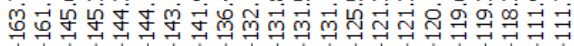

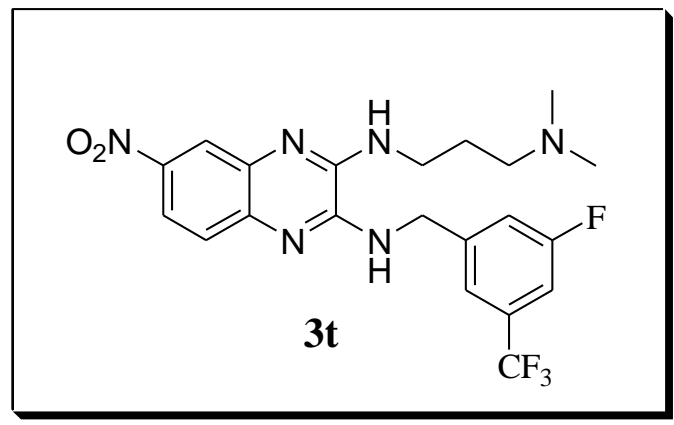

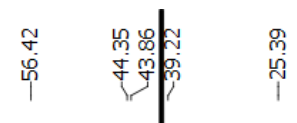

$\begin{array}{llllllllll}190 & 180 & 170 & 160 & 150 & 140 & 130 & 120 & 110 & 100 \\ \mathrm{f} 1(\mathrm{ppm})\end{array}$ 
${ }^{1} \mathrm{H}$ NMR of $3 \mathbf{u}\left(\mathrm{CDCl}_{3}, 600 \mathrm{MHz}\right)$ :-

RP-BiCFI CD3OD in CDCl3 600MHz
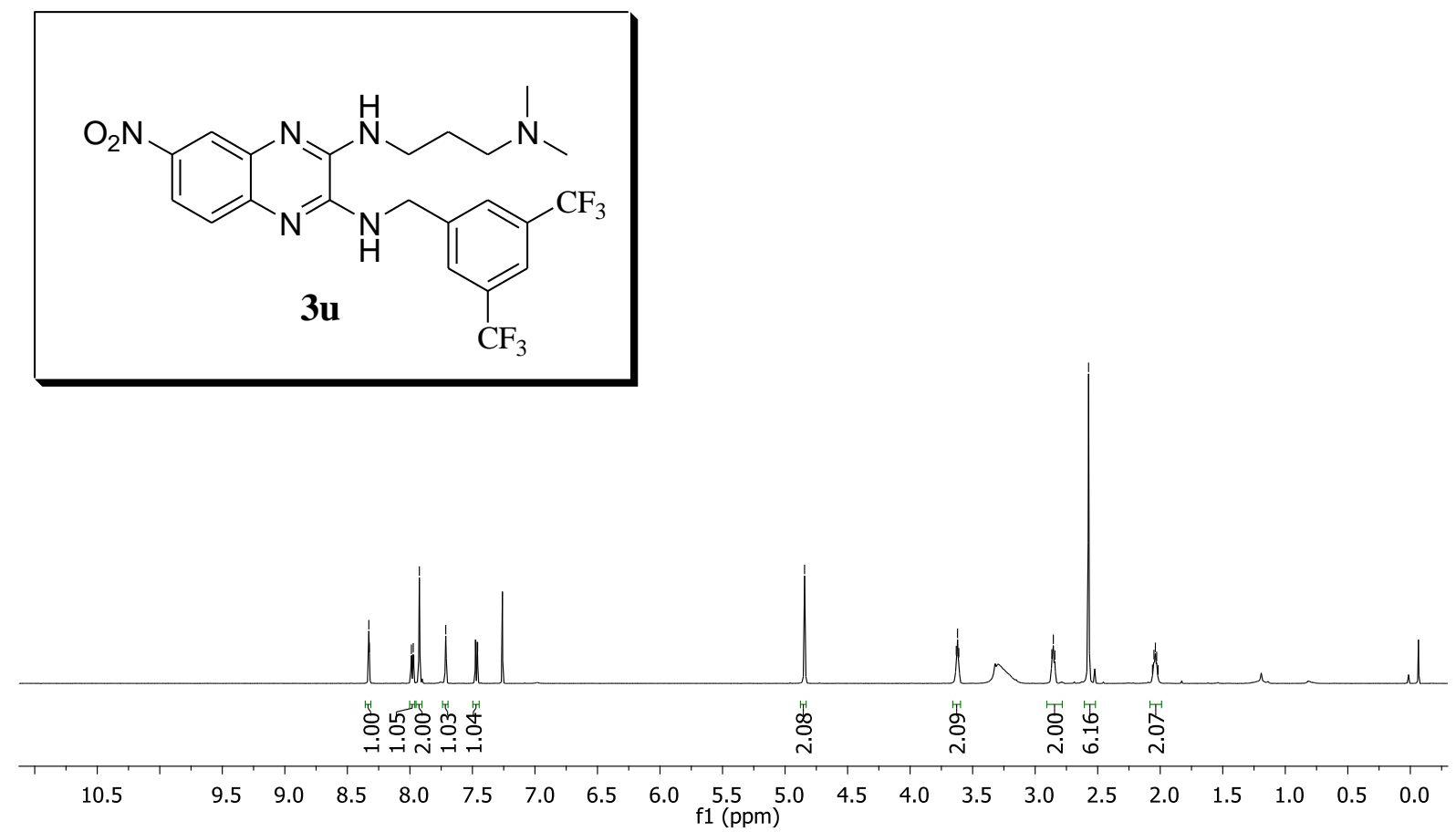

${ }^{13} \mathrm{C}$ NMR of $3 \mathbf{u}\left(\mathrm{DMSO}_{\mathrm{d}}, 150 \mathrm{MHz}\right)$ :-

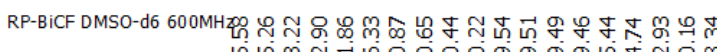

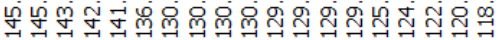

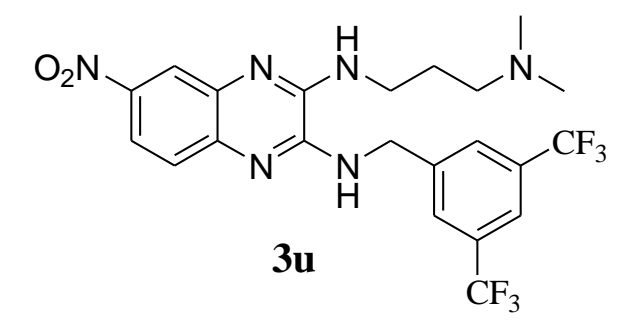

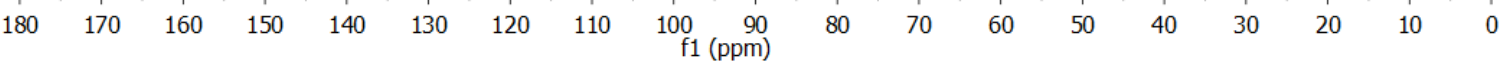


Section S2 : HPLC Chromatogram

Chromatogram of $\mathbf{3 a}$ :-

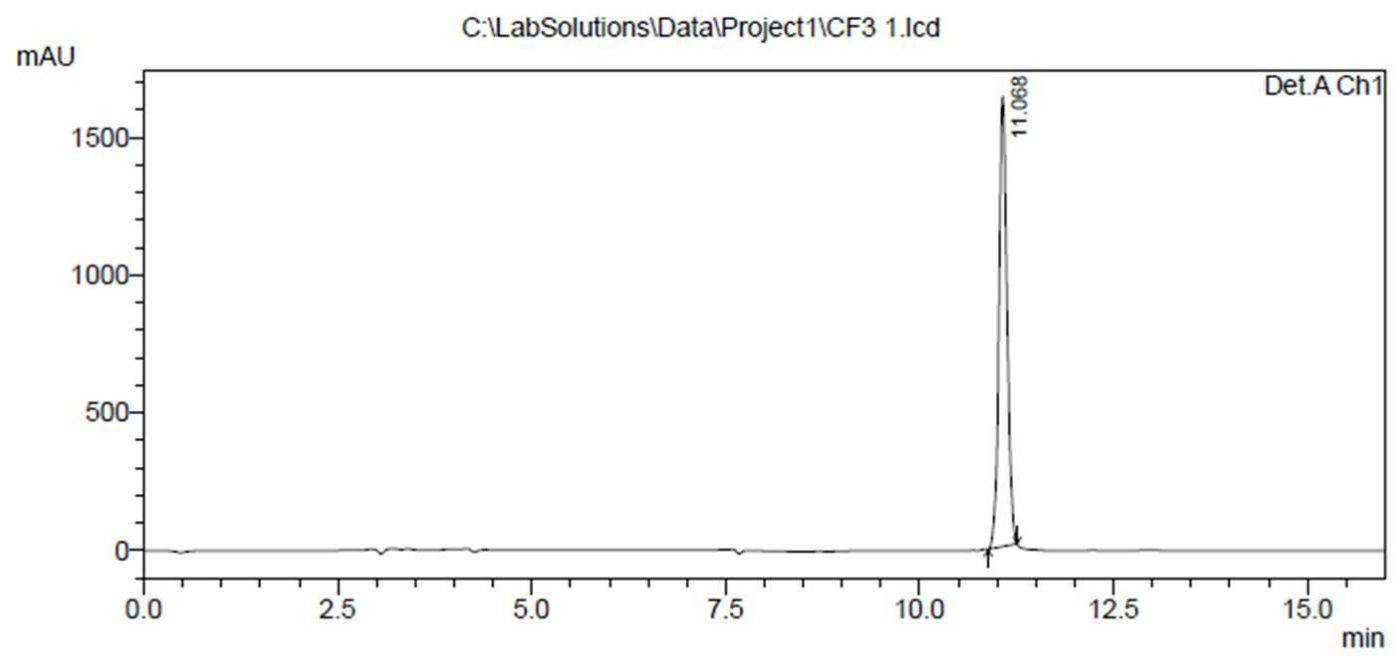

PeakTable

Detector A Ch1 $254 \mathrm{~nm}$
\begin{tabular}{|r|r|r|r|r|}
\hline Peak\# & Ret. Time & Area & \multicolumn{1}{|c|}{ Height } & \multicolumn{1}{|c|}{ Area $\%$} \\
\hline 1 & 11.068 & 11896063 & 1633629 & 100.000 \\
\hline Total & & 11896063 & 1633629 & 100.000 \\
\hline
\end{tabular}

\section{Chromatogram of $\mathbf{3 b}$ :-}

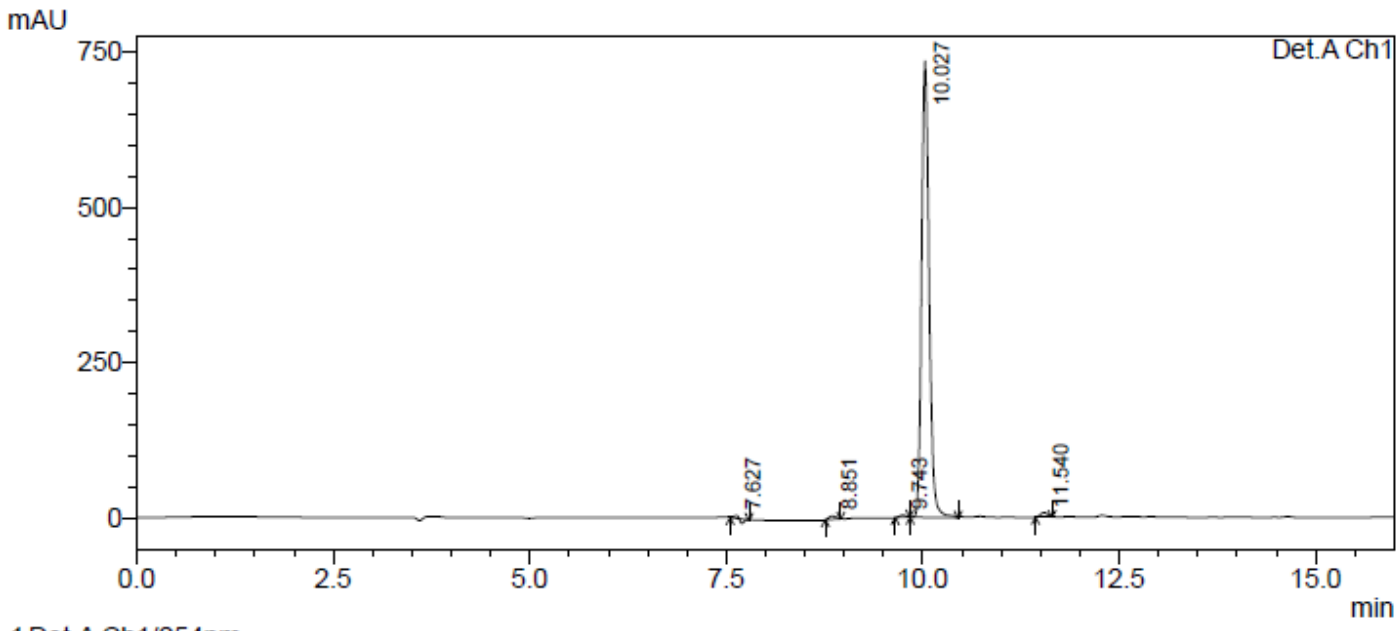

1 Det.A Ch1/254nm

\section{PeakTable}

Detector A Ch1 254nm
\begin{tabular}{|r|r|r|r|}
\hline \multicolumn{1}{|c|}{ Peak\# } & Ret. Time & \multicolumn{1}{c|}{ Area } & \multicolumn{1}{c|}{ Area $\%$} \\
\hline 1 & 7.627 & -4556 & -0.092 \\
\hline 2 & 8.851 & 34804 & 0.699 \\
\hline 3 & 9.743 & 15467 & 0.311 \\
\hline 4 & 10.027 & 4882263 & 98.100 \\
\hline 5 & 11.540 & 48842 & 0.981 \\
\hline Total & & 4976819 & 100.000 \\
\hline
\end{tabular}


Chromatogram of $\mathbf{3 c}$ :-

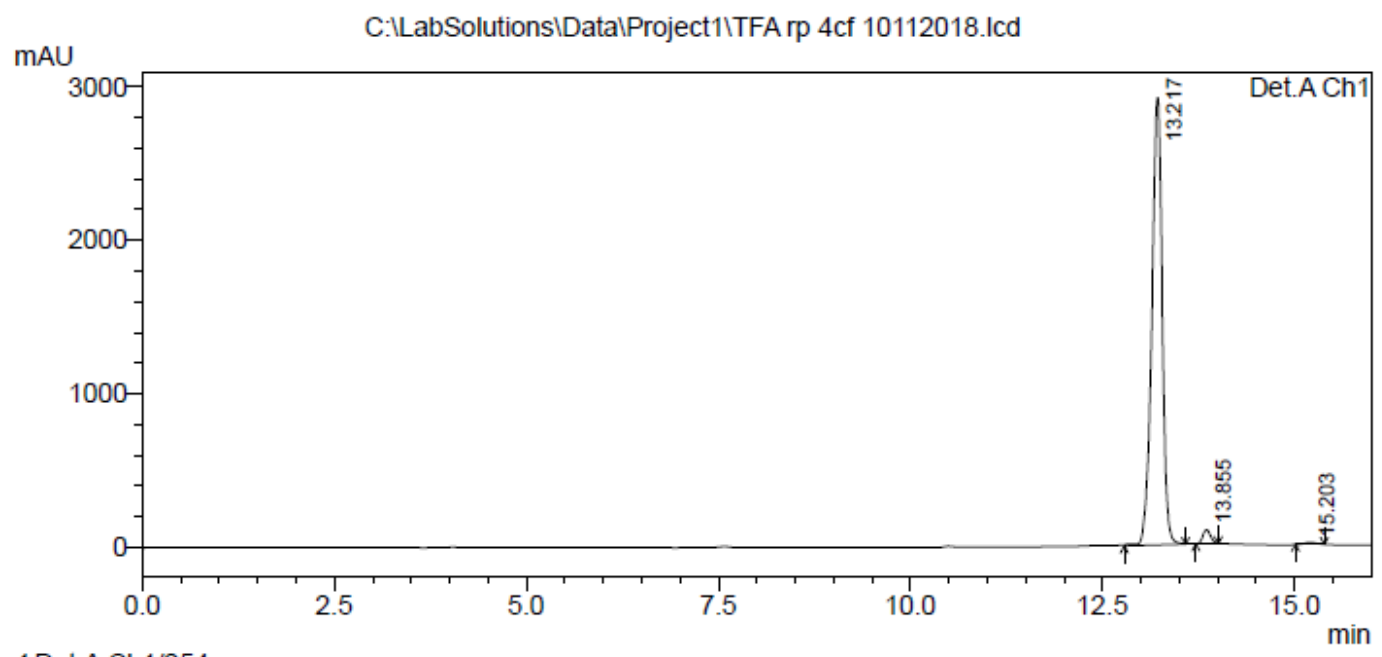

1Det.A Ch1/254nm

PeakTable
Detector A Ch1 254nm
\begin{tabular}{|r|r|r|r|}
\hline Peak\# & Ret. Time & \multicolumn{1}{c|}{ Area } & \multicolumn{1}{c|}{ Area \% } \\
\hline 1 & 13.217 & 27471554 & 97.333 \\
\hline 2 & 13.855 & 643256 & 2.279 \\
\hline 3 & 15.203 & 109367 & 0.387 \\
\hline Total & & 28224177 & 100.000 \\
\hline
\end{tabular}

\section{Chromatogram of $\mathbf{3 d}$ :-}

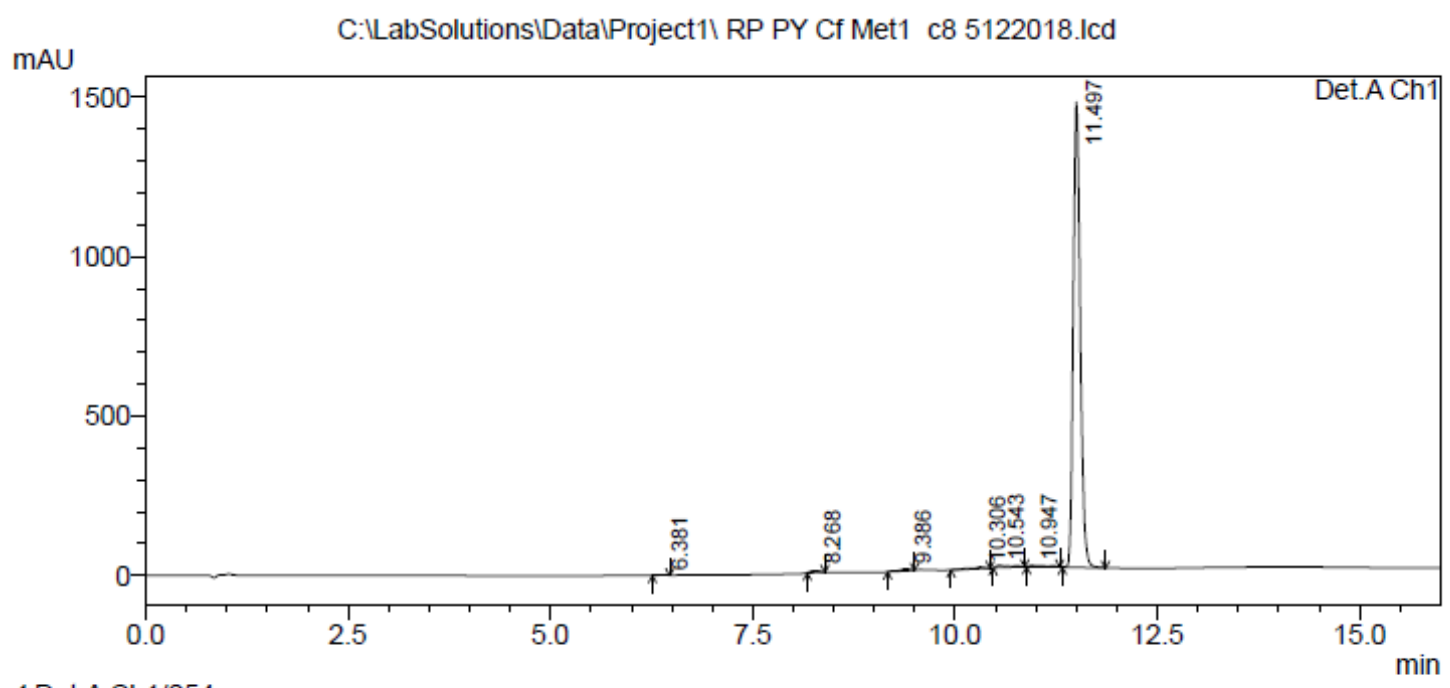

1Det.A Ch1/254nm

PeakTable

Detector A Ch $1254 \mathrm{~nm}$
\begin{tabular}{|r|r|r|r|}
\hline \multicolumn{1}{|c|}{ Peak\# } & Ret. Time & \multicolumn{1}{c|}{ Area } & \multicolumn{1}{c|}{ Area $\%$} \\
\hline 1 & 6.381 & 16529 & 0.183 \\
\hline 2 & 8.268 & 49299 & 0.545 \\
\hline 3 & 9.386 & 58100 & 0.643 \\
\hline 4 & 10.306 & 52853 & 0.585 \\
\hline 5 & 10.543 & 73645 & 0.815 \\
\hline 6 & 10.947 & 48060 & 0.532 \\
\hline 7 & 11.497 & 8742080 & 96.698 \\
\hline Total & & 9040567 & 100.000 \\
\hline
\end{tabular}


Chromatogram of $\mathbf{3 e}$ :-

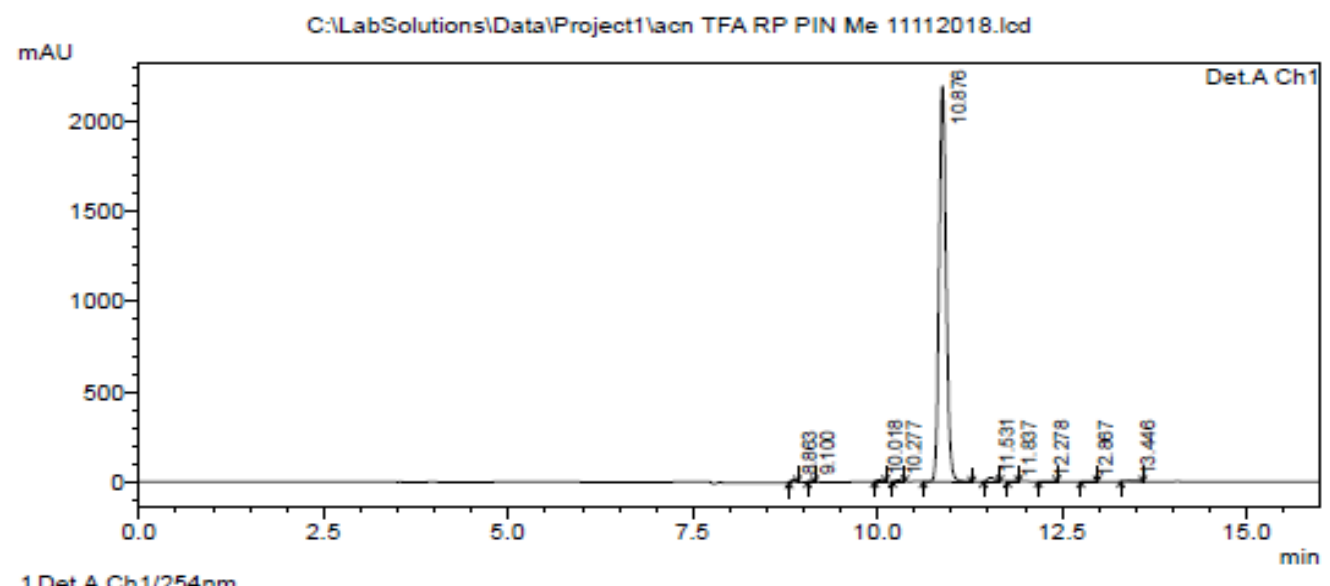

Detector A Ch1 $254 \mathrm{~nm}$
\begin{tabular}{|r|r|r|r|}
\hline \multicolumn{1}{|c|}{ Peak\# } & Ret. Time & \multicolumn{1}{c|}{ Area } & Area $\%$ \\
\hline 1 & 8.863 & 50453 & 0.337 \\
\hline 2 & 9.100 & 26349 & 0.176 \\
\hline 3 & 10.018 & 46632 & 0.312 \\
\hline 4 & 10.277 & 40286 & 0.269 \\
\hline 5 & 10.876 & 14546594 & 97.217 \\
\hline 6 & 11.531 & 136845 & 0.915 \\
\hline 7 & 11.837 & 15801 & 0.106 \\
\hline 8 & 12.278 & 6476 & 0.043 \\
\hline 9 & 12.867 & 35692 & 0.239 \\
\hline 10 & 13.446 & 57861 & 0.387 \\
\hline Total & & 14962990 & 100.000 \\
\hline
\end{tabular}

Chromatogram of $\mathbf{3 f}$ :-

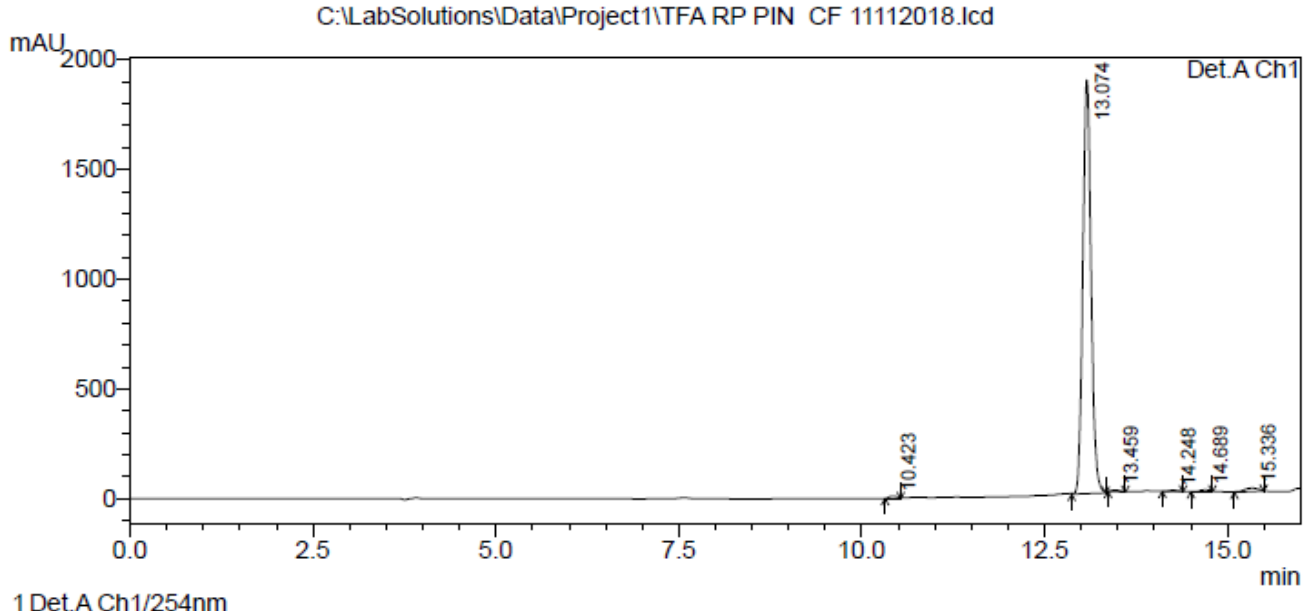

1 Det.A Ch1/254nm

PeakTable

Detector A Ch1 $254 \mathrm{~nm}$
\begin{tabular}{|r|r|r|r|}
\hline Peak\# & Ret. Time & \multicolumn{1}{c|}{ Area } & Area $\%$ \\
\hline 1 & 10.423 & 93628 & 0.614 \\
\hline 2 & 13.074 & 14758885 & 96.786 \\
\hline 3 & 13.459 & 58051 & 0.381 \\
\hline 4 & 14.248 & 54617 & 0.358 \\
\hline 5 & 14.689 & 73670 & 0.483 \\
\hline 6 & 15.336 & 210212 & 1.379 \\
\hline Total & & 15249063 & 100.000 \\
\hline
\end{tabular}


Chromatogram of $\mathbf{3 h}$ :-

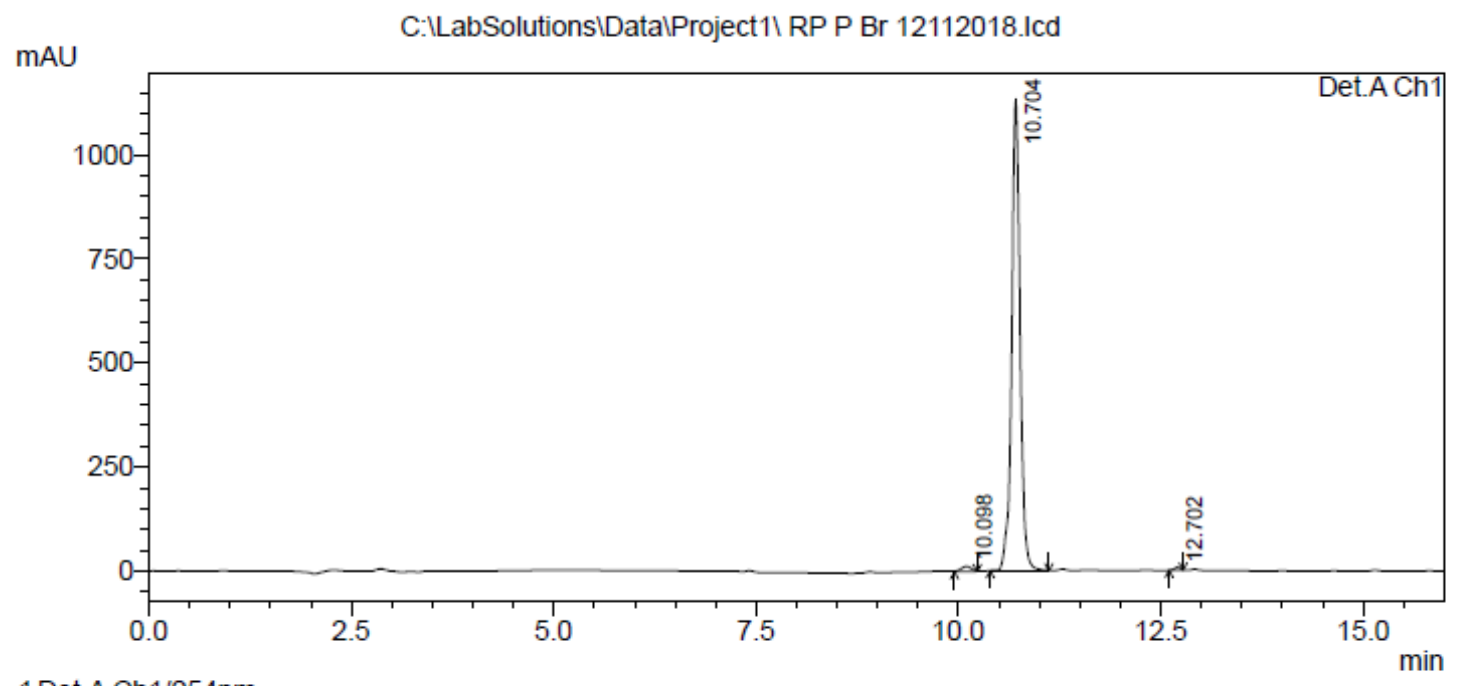

1 Det.A Ch1/254nm

PeakTable
Detector A Ch1 254nm
\begin{tabular}{|r|r|r|r|}
\hline Peak\# & Ret. Time & \multicolumn{1}{c|}{ Area } & \multicolumn{1}{c|}{ Area \% } \\
\hline 1 & 10.098 & 107115 & 1.284 \\
\hline 2 & 10.704 & 8208617 & 98.366 \\
\hline 3 & 12.702 & 29252 & 0.351 \\
\hline Total & & 8344985 & 100.000 \\
\hline
\end{tabular}

\section{Chromatogram of $\mathbf{3 i}$ :-}

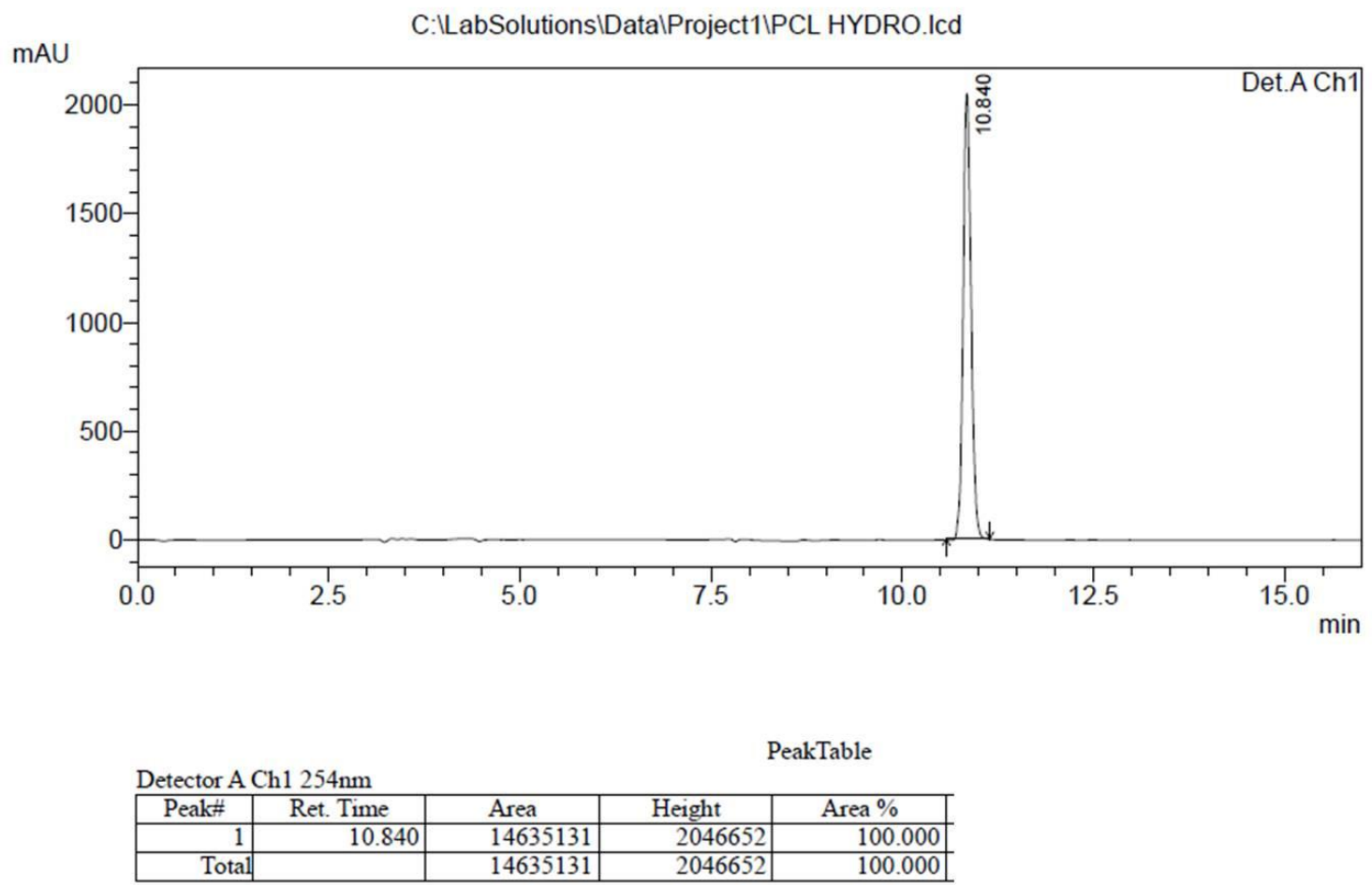


Chromatogram of $\mathbf{3 j}$ :-

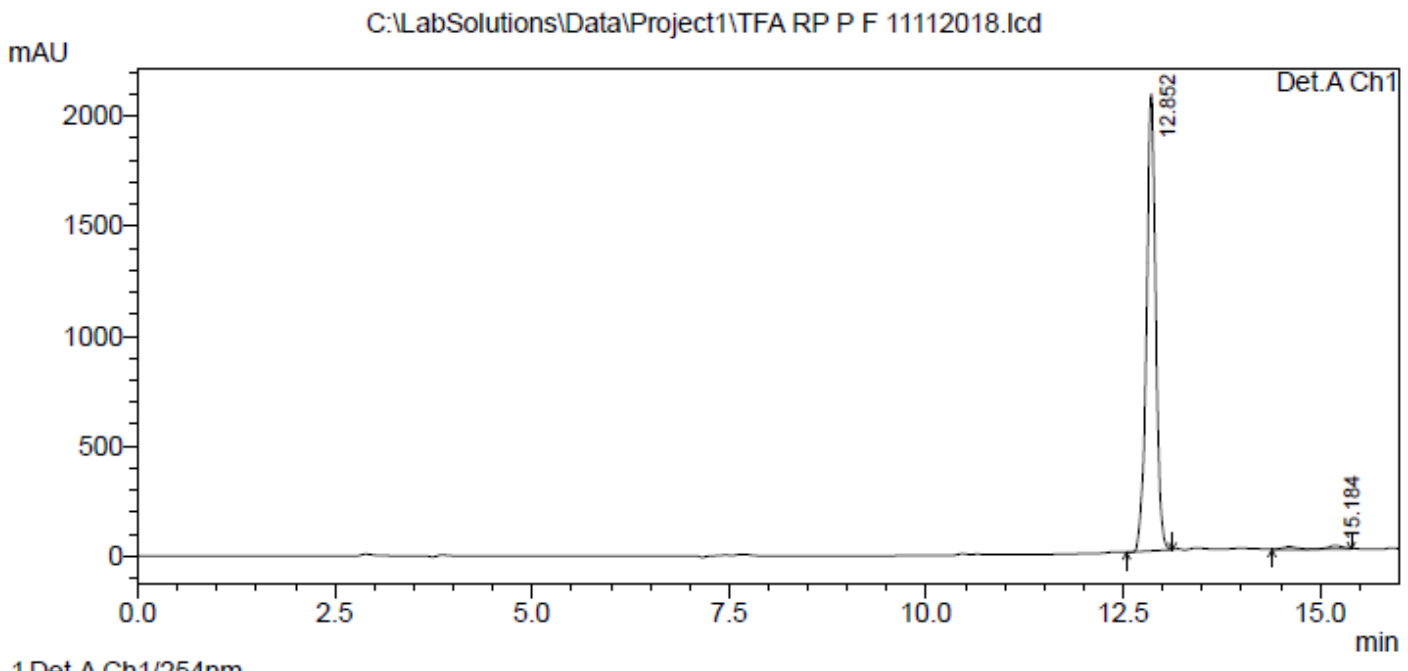

1Det.A Ch1/254nm

Detector A Ch1 254nm

PeakTable

\begin{tabular}{|r|r|r|r|}
\hline \multicolumn{1}{|c|}{ Peak\# } & Ret. Time & \multicolumn{1}{|c|}{ Area } & \multicolumn{1}{c|}{ Area \% } \\
\hline 1 & 12.852 & 16928009 & 97.605 \\
\hline 2 & 15.184 & 415381 & 2.395 \\
\hline Total & & 17343390 & 100.000 \\
\hline
\end{tabular}

Chromatogram of $\mathbf{3 k}$ :-

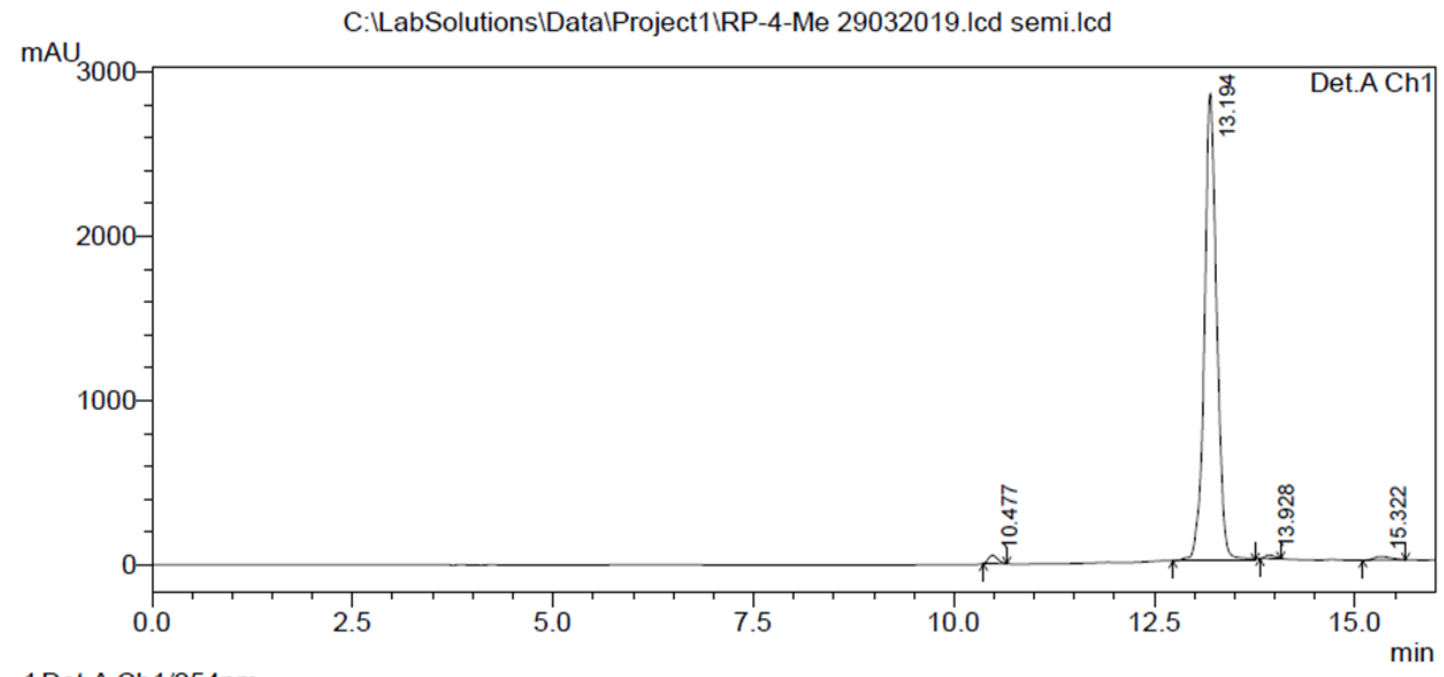

1Det.A Ch1/254nm

PeakTable

Detector A Ch $1254 \mathrm{~nm}$
\begin{tabular}{|r|r|r|r|}
\hline \multicolumn{1}{|c|}{ Peak\# } & Ret. Time & \multicolumn{1}{|c|}{ Area } & Area $\%$ \\
\hline 1 & 10.477 & 412967 & 1.323 \\
\hline 2 & 13.194 & 30322038 & 97.108 \\
\hline 3 & 13.928 & 183036 & 0.586 \\
\hline 4 & 15.322 & 307080 & 0.983 \\
\hline Total & & 31225121 & 100.000 \\
\hline
\end{tabular}


Chromatogram of $\mathbf{3 1}$ :-

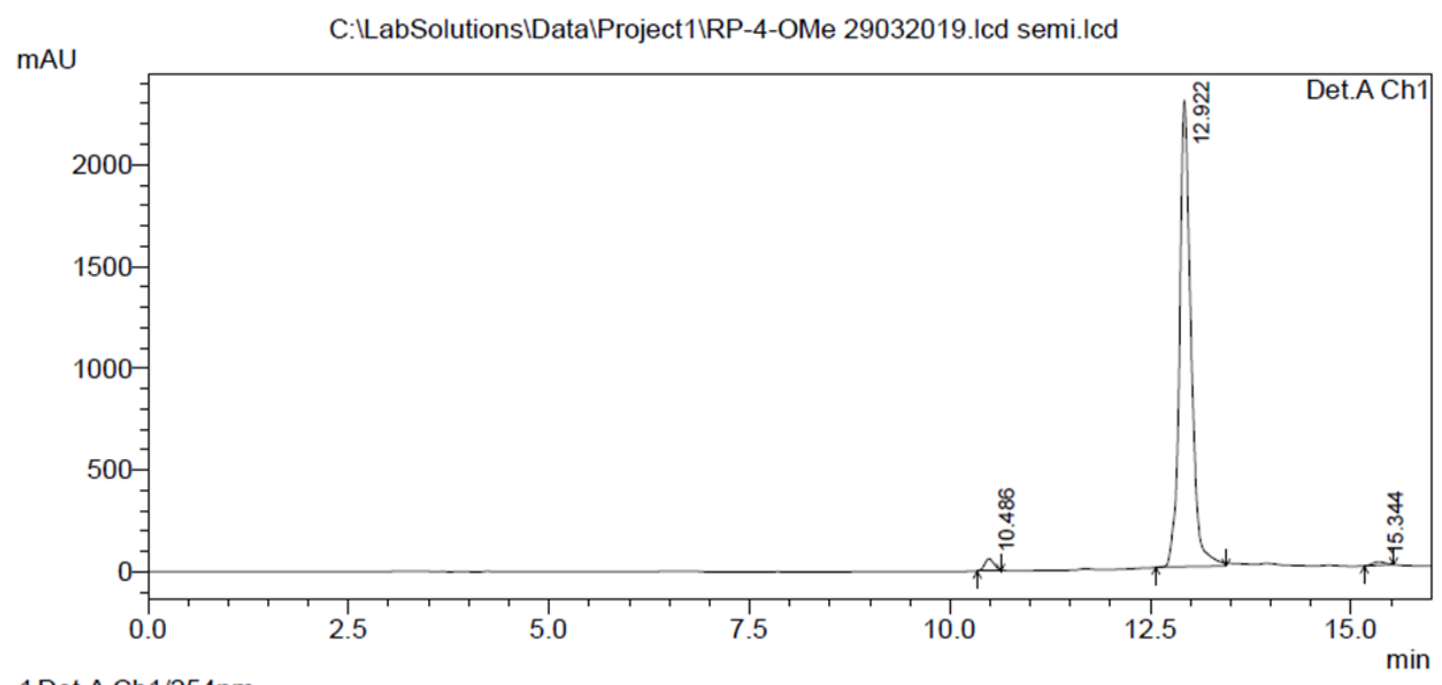

1Det.A Ch1/254nm

PeakTable

Detector A Ch1 $254 \mathrm{~nm}$
\begin{tabular}{|r|r|r|r|}
\hline Peak\# & Ret. Time & \multicolumn{1}{|c|}{ Area } & Area $\%$ \\
\hline 1 & 10.486 & 518196 & 2.200 \\
\hline 2 & 12.922 & 22841427 & 96.960 \\
\hline 3 & 15.344 & 197945 & 0.840 \\
\hline Total & & 23557569 & 100.000 \\
\hline
\end{tabular}

\section{Chromatogram of $\mathbf{3 m}$ :-}

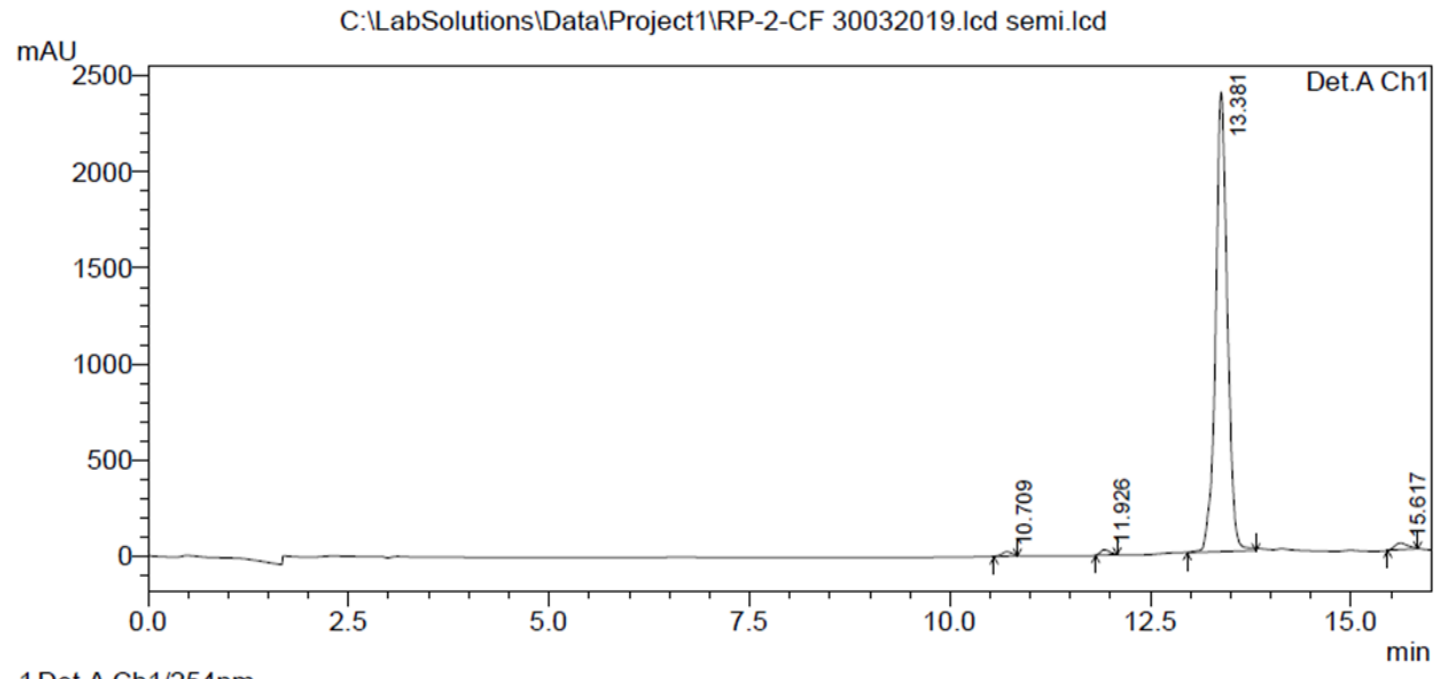

1 Det.A Ch1/254nm

PeakTable

Detector A Ch1 254nm
\begin{tabular}{|r|r|r|r|}
\hline \multicolumn{1}{|c|}{ Peak\# } & Ret. Time & \multicolumn{1}{c|}{ Area } & \multicolumn{1}{c|}{ Area $\%$} \\
\hline 1 & 10.709 & 209123 & 0.828 \\
\hline 2 & 11.926 & 218447 & 0.865 \\
\hline 3 & 13.381 & 24443799 & 96.746 \\
\hline 4 & 15.617 & 394537 & 1.562 \\
\hline Total & & 25265905 & 100.000 \\
\hline
\end{tabular}


Chromatogram of $\mathbf{3 n}$ :-

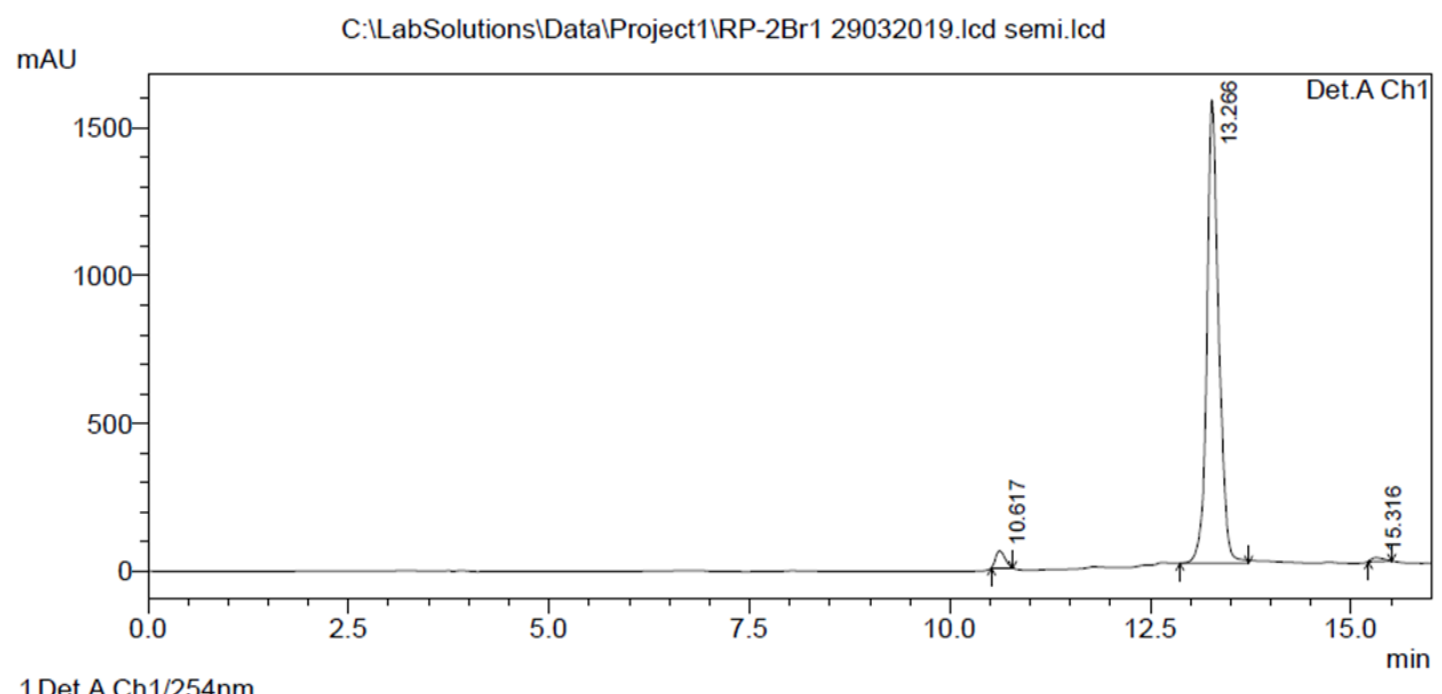

1Det.A Ch1/254nm

PeakTable

Detector A Ch1 $254 \mathrm{~nm}$
\begin{tabular}{|r|r|r|r|}
\hline \multicolumn{1}{|c|}{ Peak\# } & Ret. Time & \multicolumn{1}{|c|}{ Area } & \multicolumn{1}{c|}{ Area $\%$} \\
\hline 1 & 10.617 & 471271 & 2.826 \\
\hline 2 & 13.266 & 16059498 & 96.313 \\
\hline 3 & 15.316 & 143565 & 0.861 \\
\hline Total & & 16674334 & 100.000 \\
\hline
\end{tabular}

Chromatogram of 3o:-

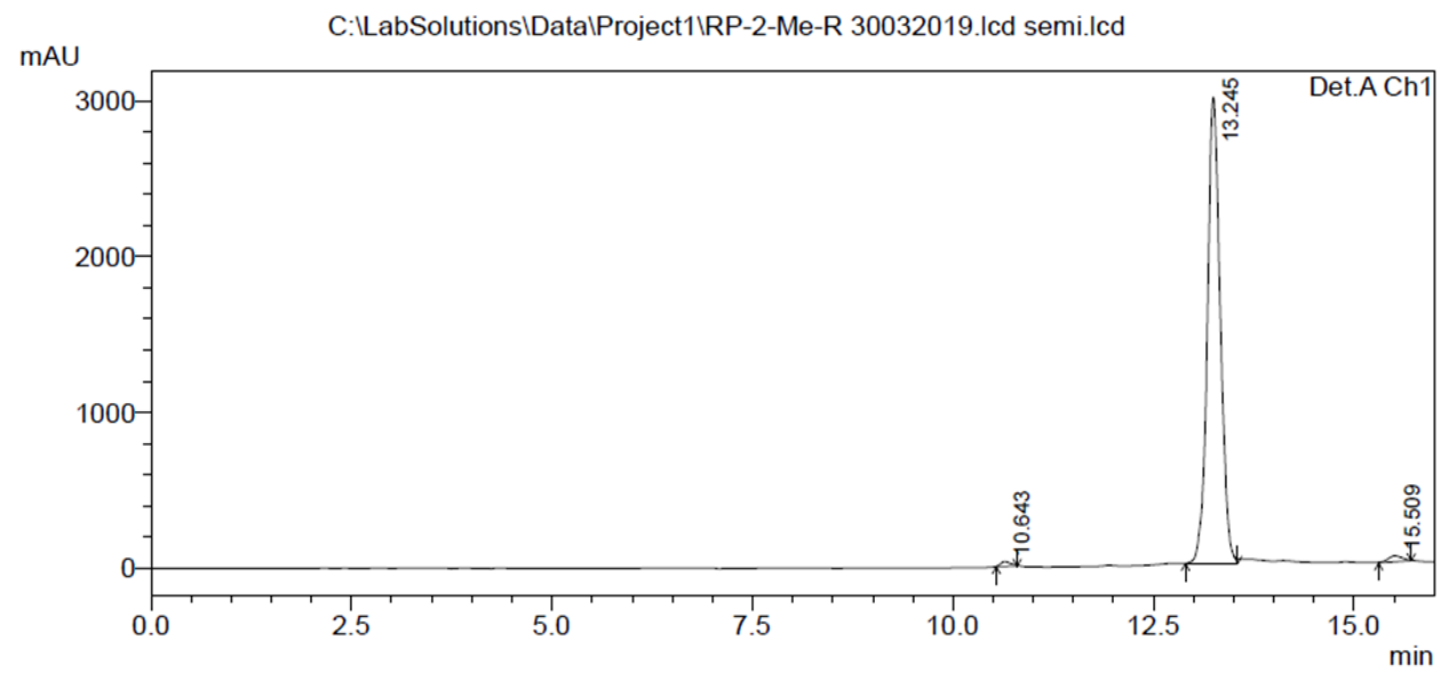

1Det.A Ch1/254nm

PeakTable

Detector A Ch1 $254 \mathrm{~nm}$
\begin{tabular}{|r|r|r|r|}
\hline Peak\# & Ret. Time & \multicolumn{1}{c|}{ Area } & Area $\%$ \\
\hline 1 & 10.643 & 263181 & 0.768 \\
\hline 2 & 13.245 & 33516853 & 97.838 \\
\hline 3 & 15.509 & 477567 & 1.394 \\
\hline Total & & 34257601 & 100.000 \\
\hline
\end{tabular}


Chromatogram of $\mathbf{3 p}$ :-

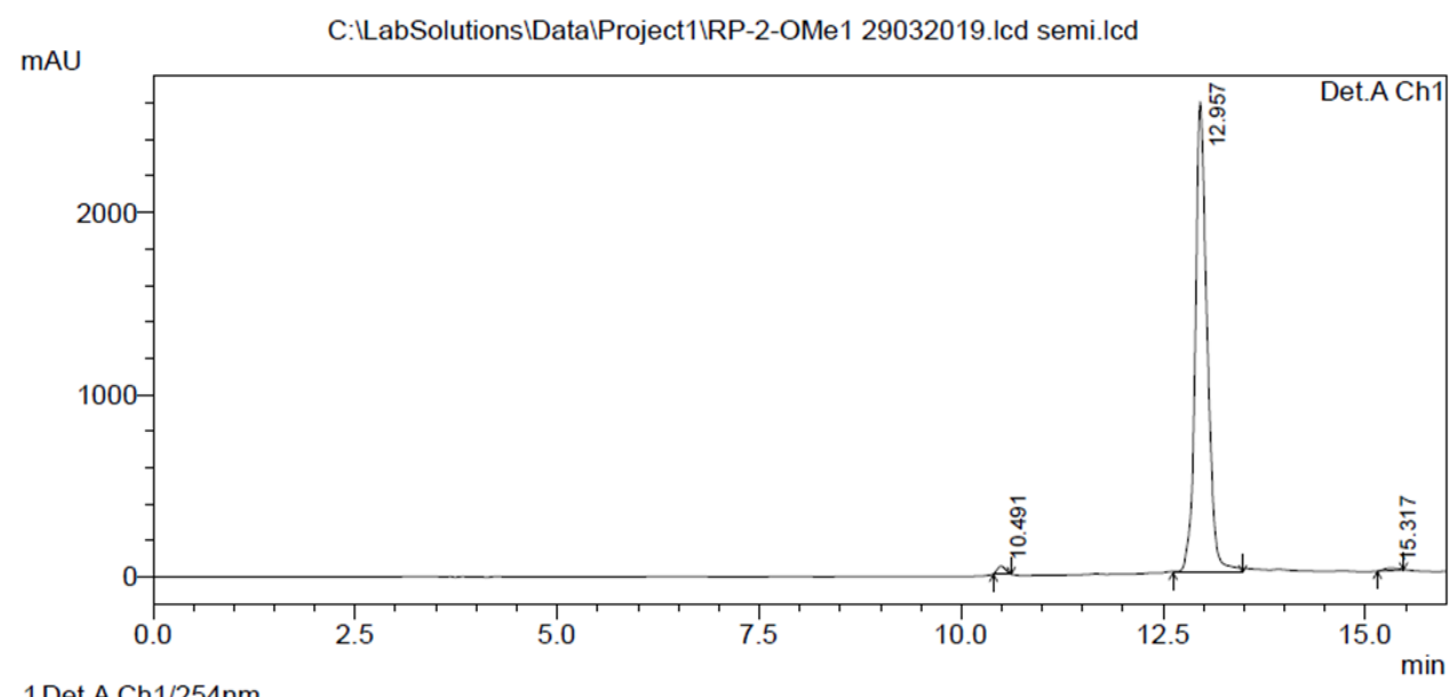

1Det.A Ch1/254nm

PeakTable

Detector A Ch1 $254 \mathrm{~nm}$
\begin{tabular}{|r|r|r|r|}
\hline \multicolumn{1}{|c|}{ Peak\# } & Ret. Time & \multicolumn{1}{|c|}{ Area } & \multicolumn{1}{c|}{ Area $\%$} \\
\hline 1 & 10.491 & 313747 & 1.161 \\
\hline 2 & 12.957 & 26562188 & 98.254 \\
\hline 3 & 15.317 & 158315 & 0.586 \\
\hline Total & & 27034250 & 100.000 \\
\hline
\end{tabular}

\section{Chromatogram of $\mathbf{3 q}$ :-}

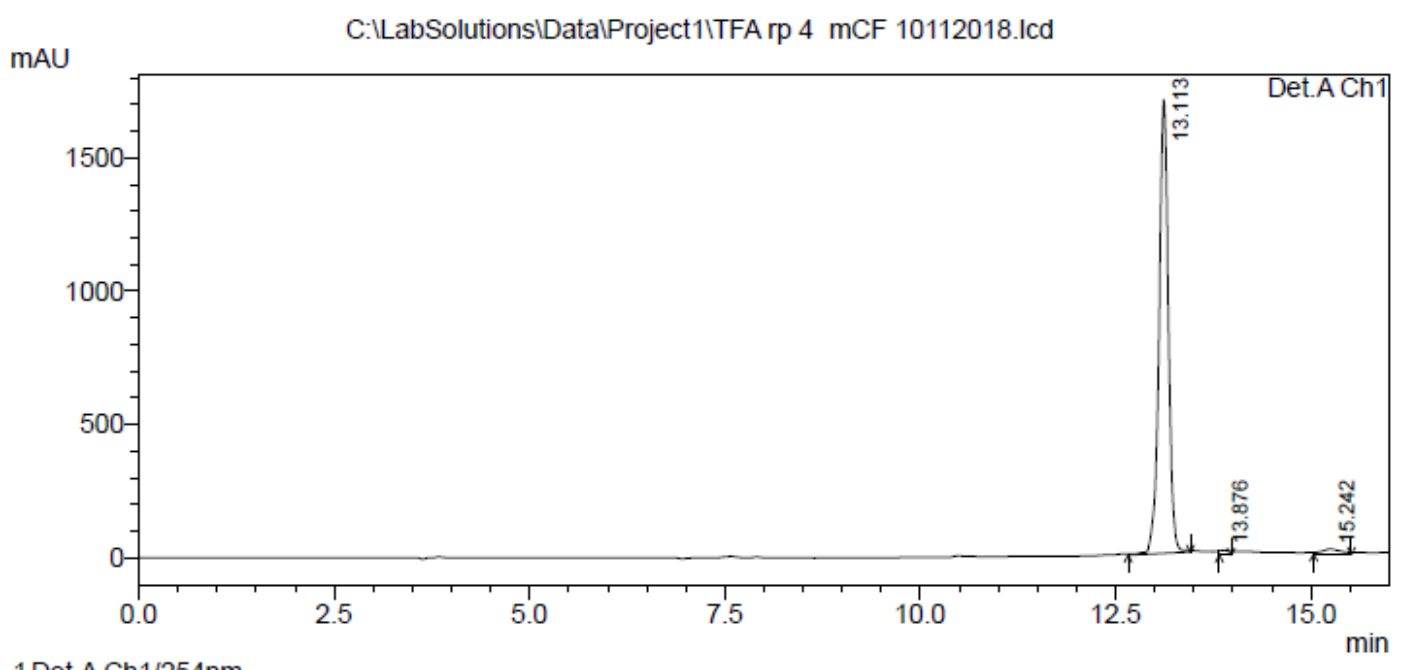

1 Det.A Ch1/254nm

PeakTable

Detector A Ch1 $254 \mathrm{~nm}$
\begin{tabular}{|r|r|r|r|}
\hline \multicolumn{1}{|c|}{ Peak\# } & Ret. Time & \multicolumn{1}{c|}{ Area } & \multicolumn{1}{c|}{ Area \% } \\
\hline 1 & 13.113 & 14140187 & 96.731 \\
\hline 2 & 13.876 & 150499 & 1.030 \\
\hline 3 & 15.242 & 327418 & 2.240 \\
\hline Total & & 14618103 & 100.000 \\
\hline
\end{tabular}


Chromatogram of $\mathbf{3 r}$ :-

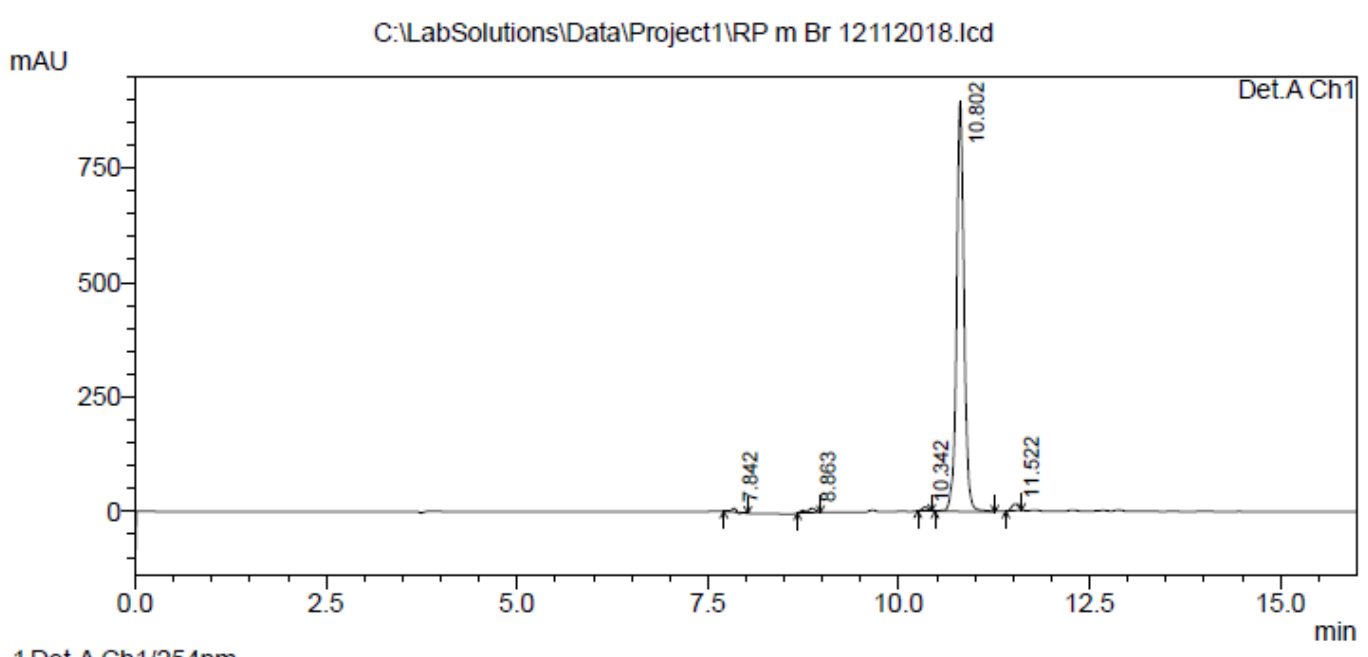

1Det.A Ch1/254nm

Detector A Ch1 254nm
\begin{tabular}{|r|r|r|r|}
\hline \multicolumn{1}{|c|}{ Peak\# } & Ret. Time & \multicolumn{1}{c|}{ Area } & \multicolumn{1}{c|}{ Area \% } \\
\hline 1 & 7.842 & 25235 & 0.400 \\
\hline 2 & 8.863 & 78694 & 1.247 \\
\hline 3 & 10.342 & 41498 & 0.658 \\
\hline 4 & 10.802 & 6073796 & 96.280 \\
\hline 5 & 11.522 & 89254 & 1.415 \\
\hline Total & & 6308478 & 100.000 \\
\hline
\end{tabular}

Chromatogram of 3s:-

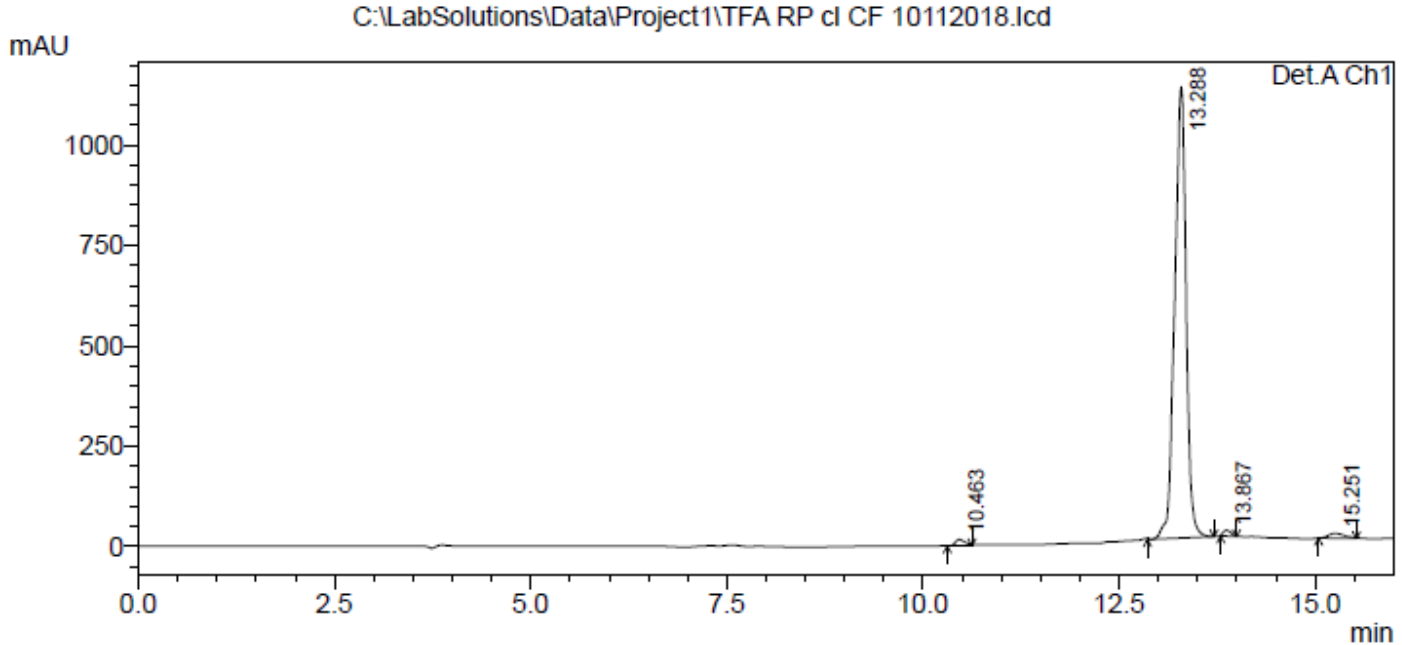

1 Det.A Ch1/254nm

PeakTable

Detector A Ch1 254nm
\begin{tabular}{|r|r|r|r|}
\hline Peak\# & Ret. Time & \multicolumn{1}{c|}{ Area } & Area $\%$ \\
\hline 1 & 10.463 & 139135 & 1.180 \\
\hline 2 & 13.288 & 11387409 & 96.554 \\
\hline 3 & 13.867 & 102093 & 0.866 \\
\hline 4 & 15.251 & 165174 & 1.401 \\
\hline Total & & 11793811 & 100.000 \\
\hline
\end{tabular}


Chromatogram of $\mathbf{3 t}$ :-

$\mathrm{mV}$

C:ILabSolutionsIDatalProject1\RP F Cf Met13 c8 5122018.Icd

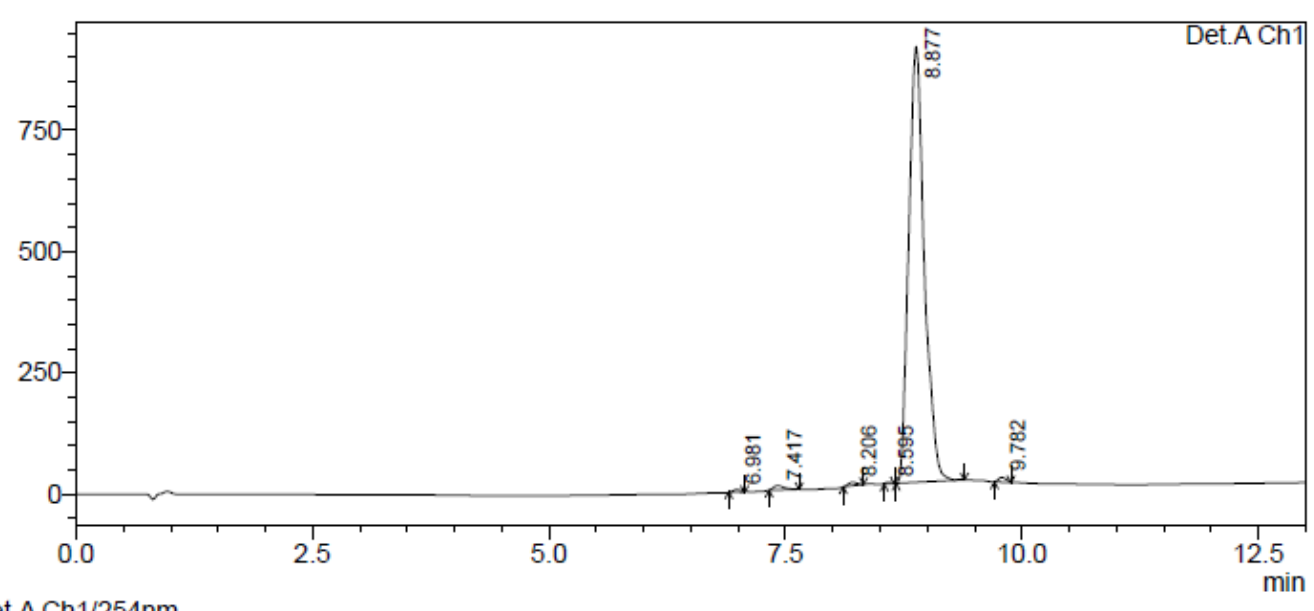

1Det.A Ch1/254nm

PeakTable
Detector A Ch1 254nm
\begin{tabular}{|r|r|r|r|}
\hline \multicolumn{1}{|c|}{ Peak\# } & Ret. Time & \multicolumn{1}{c|}{ Area } & \multicolumn{1}{c|}{ Area $\%$} \\
\hline 1 & 6.981 & 28227 & 0.268 \\
\hline 2 & 7.417 & 77530 & 0.736 \\
\hline 3 & 8.206 & 44188 & 0.419 \\
\hline 4 & 8.595 & 16883 & 0.160 \\
\hline 5 & 8.877 & 10312099 & 97.883 \\
\hline 6 & 9.782 & 56236 & 0.534 \\
\hline Total & & 10535163 & 100.000 \\
\hline
\end{tabular}

Chromatogram of 3u:-

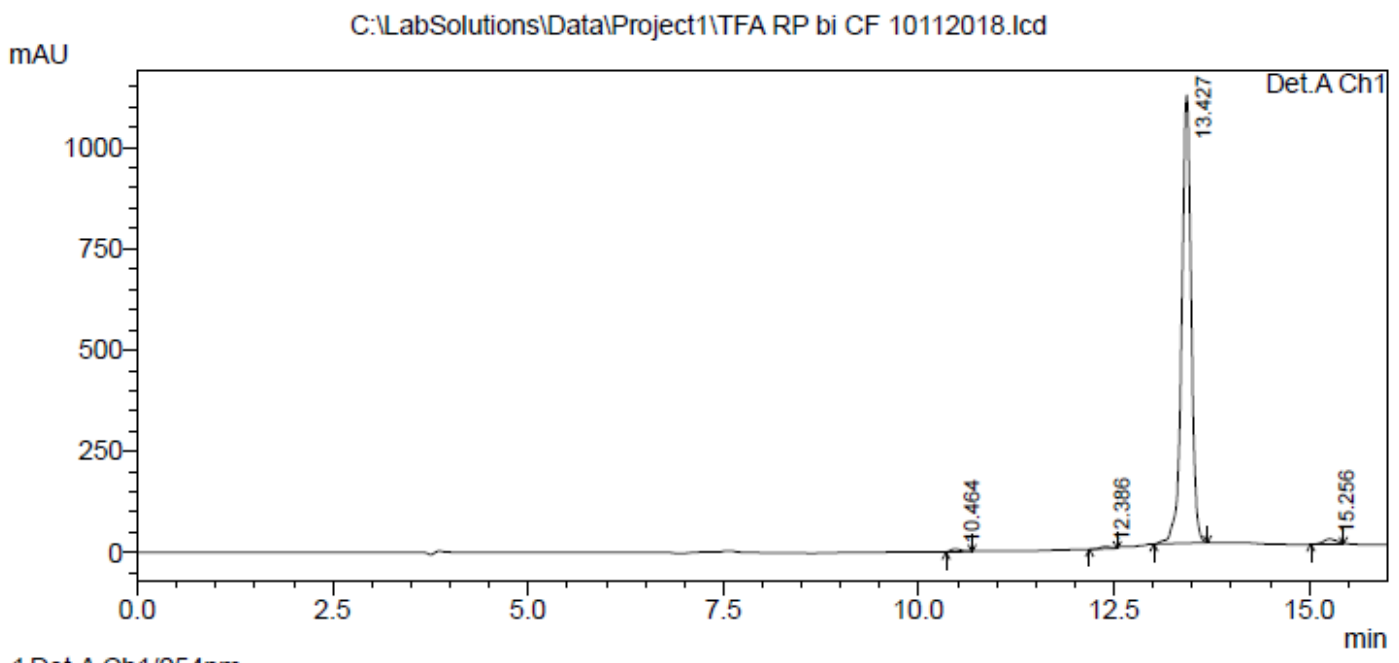

1 Det.A Ch1/254nm

PeakTable

Detector A Ch1 254nm

\begin{tabular}{|r|r|r|r|}
\hline \multicolumn{1}{|c|}{ Peak\# } & Ret. Time & \multicolumn{1}{|c|}{ Area } & \multicolumn{1}{c|}{ Area \% } \\
\hline 1 & 10.464 & 70238 & 0.732 \\
\hline 2 & 12.386 & 43369 & 0.452 \\
\hline 3 & 13.427 & 9355029 & 97.431 \\
\hline 4 & 15.256 & 133074 & 1.386 \\
\hline Total & & 9601711 & 100.000 \\
\hline
\end{tabular}


